\title{
A facile regio- and stereoselective synthesis of mannose octasaccharide of the $N$-glycan in human CD2 and mannose hexasaccharide antigenic factor $13 \mathrm{~b}$
}

\author{
Yuliang Zhu, Langqiu Chen, Fanzuo Kong* \\ Research Center for Eco-Environmental Sciences, Academia Sinica, PO Box 2871, Beijing 100085, China
}

Received 5 September 2001; accepted 14 November 2001

\begin{abstract}
A highly concise and effective synthesis of the mannose octasaccharide of the $\mathrm{N}$-linked glycan in the adhesion domain of human CD2 was achieved via TMSOTf-promoted selective 6-glycosylation of a trisaccharide 4,6-diol acceptor with a pentasaccharide donor, followed by deprotection. The pentasaccharide was constructed by selective 3,6-diglycosylation of $1,2-O$-ethylidene- $\beta$-Dmannopyranose with 2- $O$-acetyl-3,4,6-tri- $O$-benzoyl- $\alpha$-D-mannopyranosyl-( $1 \rightarrow 2)-3,4,6$-tri- $O$-benzoyl- $\alpha$-D-mannopyranosyl trichloroacetimidate, while the trisaccharide was obtained by selective 3-O-glycosylation of allyl 4,6- $O$-benzylidene- $\alpha$-D-mannopyranoside with the same disaccharide trichloroacetimidate, followed by debenzylidenation. The mannose hexasaccharide antigenic factor $13 \mathrm{~b}$ was synthesized by condensation of a trisaccharide donor, $2-O$-acetyl-3,4,6-tri- $O$-benzoyl- $\alpha$-D-mannopyranosyl- $(1 \rightarrow 2)$ 3,4,6-tri- $O$-benzoyl- $\alpha$-D-mannopyranosyl-( $1 \rightarrow 3)-4,6$-di- $O$-acetyl-2- $O$-benzoyl- $\alpha$-D-mannopyranosyl trichloroacetimidate, with a trisaccharide acceptor, methyl 3,4,6-tri- $O$-benzoyl- $\alpha$-D-mannopyranosyl-( $1 \rightarrow 2)-3,4,6$-tri- $O$-benzoyl- $\alpha$-D-mannopyranosyl- $(1 \rightarrow 2)-$ 3,4,6-tri- $O$-benzoyl- $\alpha$-D-mannopyranoside, followed by deprotection. (C) 2002 Elsevier Science Ltd. All rights reserved.
\end{abstract}

Keywords: Mannose oligosaccharides; Trichloroacetimidates; Regio- and stereoselective synthesis

\section{Introduction}

Human CD2, a cell-surface glycoprotein on $\mathrm{T}$ lymphocytes and natural killer cells, is important in mediating both cellular adhesion and signal transduction through interactions with its counterreceptor, CD58. ${ }^{1}$ The adhesion domain of CD2 bears a single $\mathrm{N}$-linked carbohydrate containing mannose oligosaccharides. ${ }^{2}$ Recny et al. indicated that glycosylation is required for human $\mathrm{CD} 2$ adhesion function. ${ }^{3}$ Moreover, transmembrane CD2 variants with mutations in the consensus N-glycosylation sequence $\mathrm{Asn}^{65}$-Gly ${ }^{66}$ $\mathrm{Thr}^{67}\left(\mathrm{~N} 65 \mathrm{Q}^{3}\right.$ or $\left.\mathrm{T}^{67 \mathrm{~A}^{4}}\right)$ that preclude attachment of

\footnotetext{
* Corresponding author. Tel.: + 86-10-62936613; fax: 8610-62923563.

E-mail address: fzkong@mail.rcees.ac.cn (F. Kong).
}

the high mannose $N$-glycan at $\mathrm{Asn}^{65}$ could be normally expressed on cell surfaces, but showed neither antibodynor ligand-binding activity. These data suggest that the $\mathrm{N}$-linked adhesion domain glycan on human CD2 plays an important role in maintaining native receptor structure. Wagner and co-workers investigated the solution structure of a fragment containing the high mannose $N$-glycan by NMR spectrometry. ${ }^{2}$ Bewley and OteroQuintero reported the important binding of anti-HIV protein cyanovirin-N to high mannose oligosaccharides very recently. ${ }^{5}$ For a study of the details of the recognition mechanism, chemically synthesized oligosaccharides are required because such homogeneous oligosaccharide samples are hardly obtainable from natural sources. We present herein a facile and convergent synthesis of the mannose octasaccharide of the $N$-glycan in the adhesion domain of human CD2. Meanwhile we also describe an efficient synthesis of mannose hexasaccharide antigenic factor $13 b^{6}$ using a similar strategy and the same synthons. 


\section{Results and discussion}

Scheme 1 shows the structure of the high-mannose $N$-glycan of human $\mathrm{CD} 2_{105}$ containing the D1 D2 isomer of Man-8 as marked with a bracket. Retrosynthetic analysis indicated that the mannose octamer can be obtained by condensation of two moieties, i.e., a mannose pentamer donor and a mannose trimer acceptor. The pentasaccharide then can be constructed from a disaccharide donor and a 1,2-ethylidenated mannose acceptor, while the trisaccharide can be built from the same disaccharide donor and another simple mannose derivative.

We previously reported a highly regio- and stereoselective method $^{7}$ through orthoester formation-rearrangement for the synthesis of oligosaccharides, and some biologically important oligosaccarides such as the phytoalexin elicitor hexasaccharide were efficiently synthesized. ${ }^{8}$ Later on we found that by controlling the reaction conditions, $(1 \rightarrow 6)$-linked oligosaccharides with 1,2-trans linkages were readily constructed with acylated glycosyl trichloroimidates as the donors and unprotected or partially protected saccharides as the acceptors through orthoester intermediates. ${ }^{9}$ We also found that $(1 \rightarrow 2)$-linked mannosyl disaccharides were easily synthesized by self-condensation of benzoylated mannose 1,2-orthoester. ${ }^{10}$ Based on these new findings, we designed a concise and effective route for the synthesis of the mannosyl octasaccharide as shown in Scheme 2.

Orthoester 3 was obtained from acetylated mannosyl bromide $\mathbf{1}$ through the intermediate $\mathbf{2}$ in high yield $(90 \%)$ by a previously reported method. ${ }^{11}$ Self-conden-
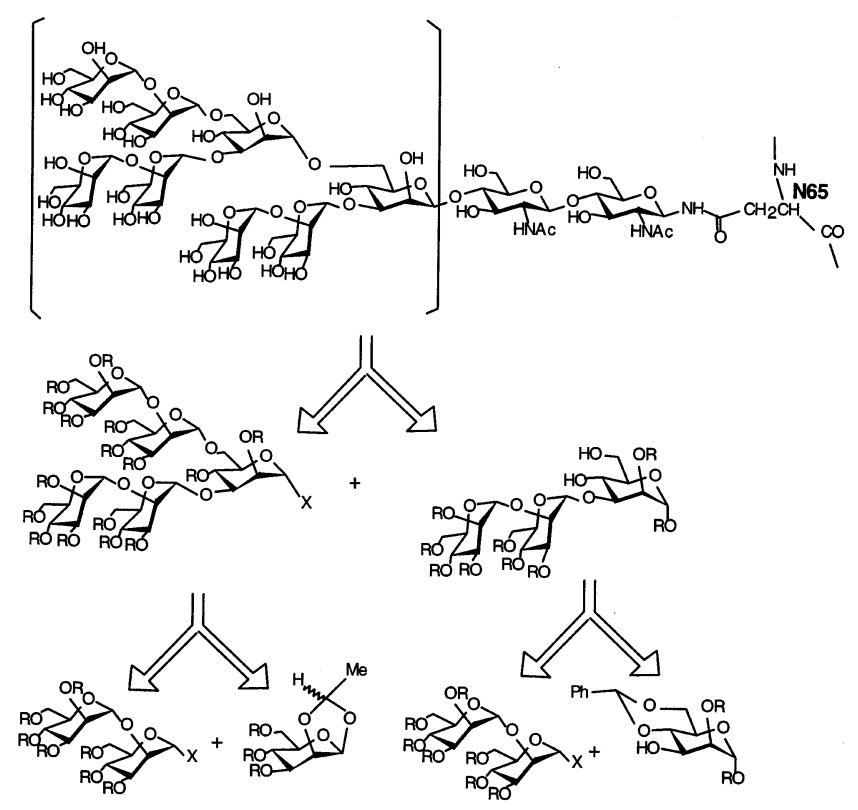

Scheme 1. sation of $\mathbf{3}$ promoted by catalytic TMSOTf gave the disaccharide 4 in satisfactory yield (66\%). Deallylation with $\mathrm{PdCl}_{2}$, followed by activation with $\mathrm{CCl}_{3} \mathrm{CN}$ in the presence of DBU or $\mathrm{K}_{2} \mathrm{CO}_{3}$, produced the disaccharide donor 5. Regioselective coupling of 5 with allyl 4,6-Obenzylidene- $\alpha$-D-mannopyranoside $(6)^{12}$ resulted in the $(1 \rightarrow 3)$-linked trisaccharide 7 , and subsequent acetylation yielded the trisaccharide 8 . The ${ }^{1} \mathrm{H}$ NMR spectrum of $\mathbf{8}$ showed $\mathrm{H}-2$ of the reducing mannose at $\delta 5.42$ ppm with $J_{1,2} 1.4$ and $J_{2,3} 2.8 \mathrm{~Hz}$, indicating the 3 -selective glycosylation of $\mathbf{6}$. Debenzylidenation of 8 gave the trisaccharide acceptor $\mathbf{9}$. For the pentasaccharide donor synthesis, 1,2- $O$-ethylidene- $\beta$-D-mannopyranose (10) was chosen as the starting material since its glycosylation has excellent regioselectivity for both the 6- and 3 -positions via orthoester intermediates, ${ }^{7 \mathrm{~b}}$ and its ethylidene group is readily removed for the next reaction. Thus, coupling of $\mathbf{5}$ (2.2 equiv) with $\mathbf{1 0}$ in dichloromethane in the presence of catalytic TMSOTf produced the pentasaccharide 11. It was noted that addition of TMSOTf was performed at $-20{ }^{\circ} \mathrm{C}$ to ensure that at the initial stage of the reaction, the orthoester was formed with high regioselectivity, ${ }^{7 b, 9}$ and then it rearranged to the required pentasaccharide along with extension of the reaction time. If TMSOTf was added at room temperature, the reaction was very fast, and the 3,4,6-trisubstituted heptasaccharide was the major product, even if only 2 equiv of 5 were used. Acetylation of $\mathbf{1 1}$ gave fully protected pentasaccharide 12, and its ${ }^{1} \mathrm{H}$ NMR spectrum showed a newly emerged triplet at $\delta 5.20 \mathrm{ppm}$ with $J_{3,4}=J_{4,5} 10 \mathrm{~Hz}$ for $\mathrm{H}-4$ confirming the 3,6-glycosylation of $\mathbf{1 0}$. Removal of the ethylidene group of $\mathbf{1 2}$ with $90 \%$ trifluoroacetic acid, followed by acetylation with acetic anhydride in pyridine, selective 1-O-deacetylation with $\left(\mathrm{NH}_{4}\right)_{2} \mathrm{CO}_{3}$ and subsequent activation with $\mathrm{CCl}_{3} \mathrm{CN}$ in the presence of DBU or $\mathrm{K}_{2} \mathrm{CO}_{3}$ afforded the pentasaccharide donor 14 . Selective 6-O-glycosylation of $\mathbf{9}^{9,13}$ with $\mathbf{1 4}$ produced the octasaccharide $\mathbf{1 5}$ as the major product. Acetylation of $\mathbf{1 5}$ gave the fully acylated octasaccharide 16, and the structures of 15 and 16 were characterized by mass spectrometry, which showed $m / z 3479\left[\mathrm{M}^{+}\right]$and 3521 $\left[\mathrm{M}^{+}\right]$, respectively. The ${ }^{13} \mathrm{C}$ NMR spectrum of $\mathbf{1 6}$ gave eight signals for C-1 from $\delta$ 96.0-99.7 ppm, with ${ }^{2} J_{\mathrm{C}-1-\mathrm{H}-1} 170-174 \mathrm{~Hz}$, indicating all $\alpha$ linkages. Deacylation was conducted at room temperature in ammonia-saturated methanol for one week giving the target mannose octasaccharide $\mathbf{1 7}$, whose bioassay is in process.

The synthesis of mannose hexasaccharide antigenic factor $13 \mathrm{~b}$ was accomplished using a similar strategy and the same synthons, as shown in Scheme 3. Thus, coupling of 2- $O$-acetyl-3,4,6-tri- $O$-benzoyl- $\alpha$-D-mannopyranosyl trichloroacetimidate $(\mathbf{1 8})^{10}$ with methyl $3,4,6-$ 

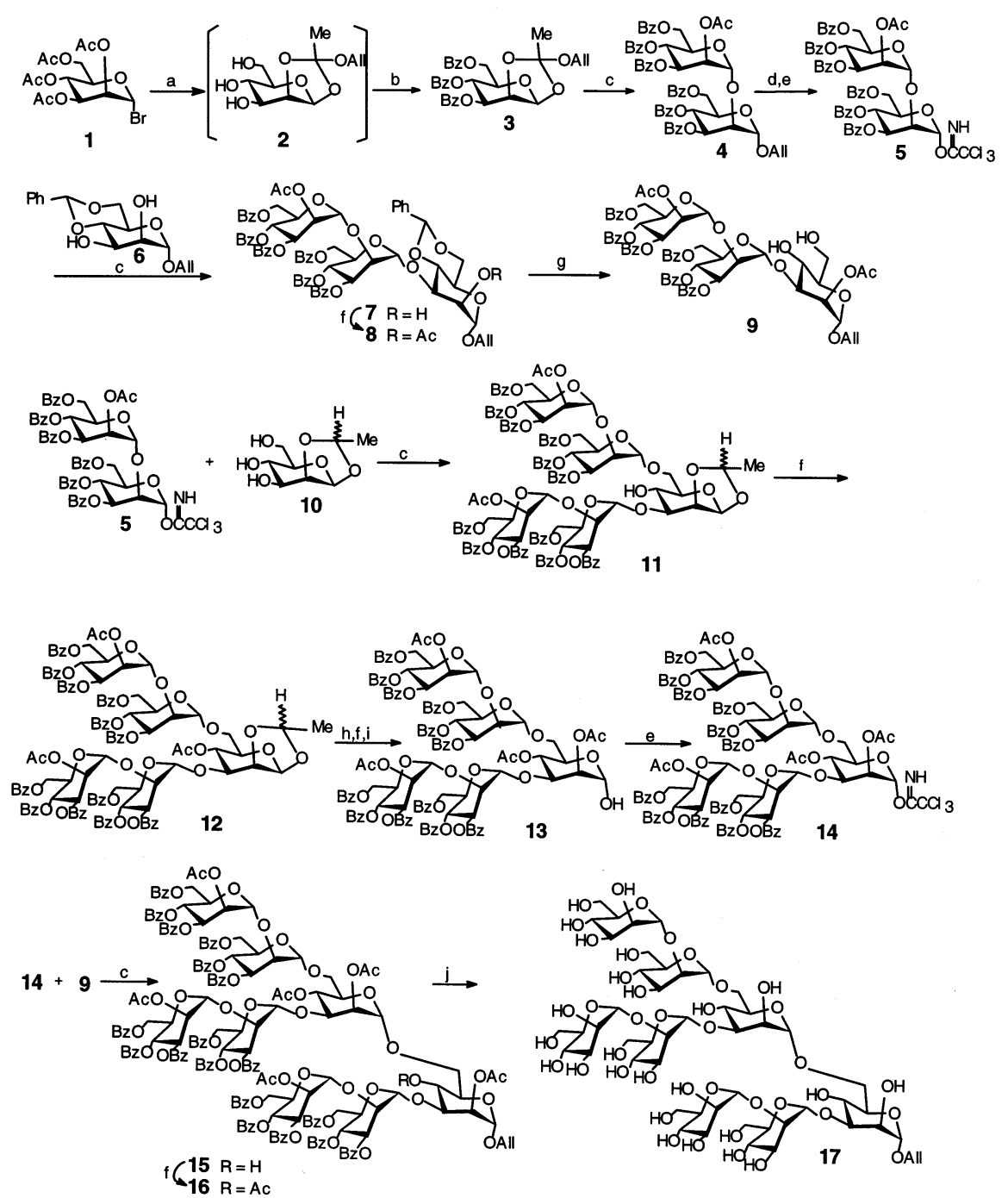

Scheme 2. Reagents: (a) lutidine, $\mathrm{CH}_{2} \mathrm{Cl}_{2}, 4 \AA \mathrm{MS}$, then $\mathrm{MeONa}, \mathrm{MeOH}$; (b) $\mathrm{BzCl}$-pyridine (dry); (c) TMSOTf, $\mathrm{CH}_{2} \mathrm{Cl}_{2}, 4 \AA \mathrm{MS}$; (d) $\mathrm{PdCl}_{2}, \mathrm{CH}_{2} \mathrm{Cl}_{2}$; (e) $\mathrm{CCl}_{3} \mathrm{CN}$, DBU, $\mathrm{CH}_{2} \mathrm{Cl}_{2}$; (f) $\mathrm{Ac}_{2} \mathrm{O}$-pyridine (dry); (g) 99.9:0.1 $\mathrm{CH}_{3} \mathrm{OH}-\mathrm{CH}_{3} \mathrm{COCl}$; (h) $90 \% \mathrm{CF}_{3} \mathrm{COOH}$; (i) DMF, $\left(\mathrm{NH}_{4}\right)_{2} \mathrm{CO}_{3} ;$ (j) $\mathrm{NH}_{3}-\mathrm{MeOH}$.

tri- $O$-benzoyl- $\alpha$-D-mannopyranosyl- $(1 \rightarrow 2)-3,4,6$-tri- $O$ benzoyl- $\alpha$-D-mannopyranoside $(\mathbf{1 9})^{10}$ gave the trisaccharide 20, and subsequent deacetylation afforded the trisaccharide acceptor $\mathbf{2 1} .^{10}$ The trisaccharide donor $\mathbf{2 6}$ was obtained from 7 through benzoylation $(\rightarrow \mathbf{2 2})$, debenzylidenation $(\rightarrow \mathbf{2 3})$, acetylation $(\rightarrow \mathbf{2 4})$, deallylation $(\rightarrow \mathbf{2 5})$, and trichloroacetimidation. Condensation of 26 with 21 yielded the hexasaccharide 27, and its deacylation gave the antigenic factor $13 \mathrm{~b}$.

In summary, we present herein a very effective regioand stereoselective synthesis of the mannose octasaccharide of the $N$-glycan in human CD2 and the mannose hexasaccharide antigenic factor $13 \mathrm{~b}$. Preparation on large scale is possible owing to the relatively simple procedure and convenient raw materials. The method described will be suitable for the preparation of higher mannose oligosaccharides with similar structure.

\section{Experimental}

General methods. - Optical rotations were determined at $25^{\circ} \mathrm{C}$ with a Perkin-Elmer model 241-MC automatic polarimeter. Melting points were determined with a 'Mel-Temp' apparatus. ${ }^{1} \mathrm{H}$ NMR, ${ }^{13} \mathrm{C}$ NMR and ${ }^{1} \mathrm{H}-{ }^{1} \mathrm{H}$ COSY and ${ }^{1} \mathrm{H}-{ }^{13} \mathrm{C}$ COSY spectra were recorded with Bruker ARX 400 spectrometers (400 $\mathrm{MHz}$ for ${ }^{1} \mathrm{H}, 100 \mathrm{MHz}$ for ${ }^{13} \mathrm{C}$ ) at $25^{\circ} \mathrm{C}$ for solutions in $\mathrm{CDCl}_{3}$ or $\mathrm{D}_{2} \mathrm{O}$ as indicated. Chemical shifts are given in ppm downfield from internal $\mathrm{Me}_{4} \mathrm{Si}$. Mass spectra were measured using MALDI-TOF-MS with CCA as matrix or recorded with a VG PLATFORM mass spectrometer using the ESI mode. IR spectra were recorded with a Hitachi 270-30 spectrometer. Thinlayer chromatography (TLC) was performed on Silica Gel $\mathrm{HF}_{254}$ with detection by charring with $30 \%(\mathrm{v} / \mathrm{v})$ $\mathrm{H}_{2} \mathrm{SO}_{4}$ in $\mathrm{MeOH}$ or in some cases by a UV lamp. 

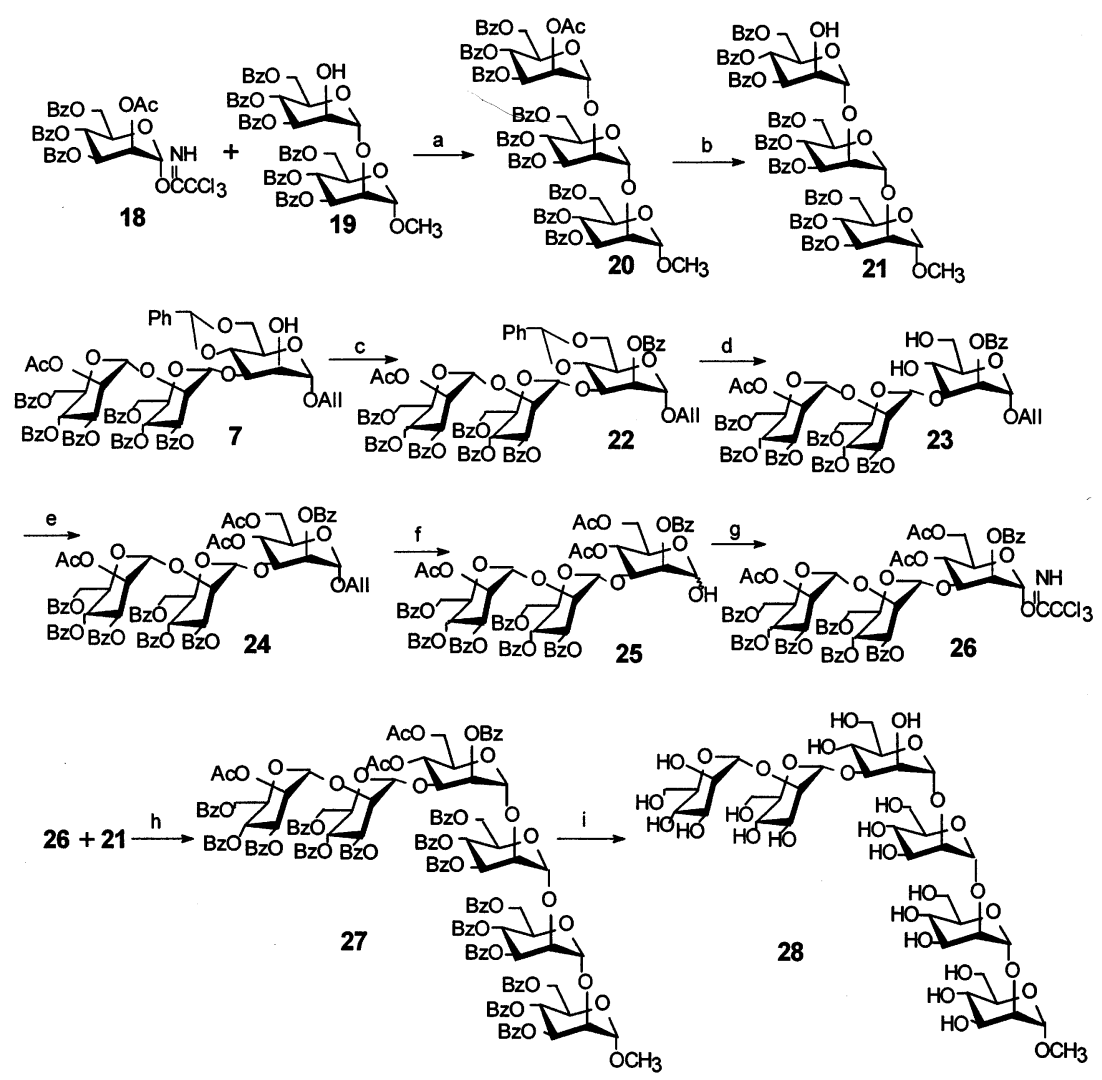

Scheme 3. Reagents: (a) TMSOTf, $\mathrm{CH}_{2} \mathrm{Cl}_{2}, 4 \AA \mathrm{AS}$; (b) 95:5 $\mathrm{CH}_{3} \mathrm{OH}-\mathrm{CH}_{3} \mathrm{COCl}$; (c) BzCl-pyridine (dry); (d) 99.9:0.1 $\mathrm{CH}_{3} \mathrm{OH}-\mathrm{CH}_{3} \mathrm{COCl}$; (e) $\mathrm{Ac}_{2} \mathrm{O}$-pyridine (dry); (f) $\mathrm{PdCl}_{2}, \mathrm{HOAc}-\mathrm{NaOAc}$; (g) $\mathrm{CCl}_{3} \mathrm{CN}, \mathrm{DBU}, \mathrm{CH}_{2} \mathrm{Cl}_{2}$; (h) TMSOTf, $\mathrm{CH}_{2} \mathrm{Cl}_{2}, 4 \AA$ MS; (i) $\mathrm{NH}_{3}-\mathrm{MeOH}$.

Column chromatography was conducted by elution of a column $(16 \times 240 \mathrm{~mm}, 18 \times 300 \mathrm{~mm}, 35 \times 400 \mathrm{~mm})$ of silica gel (100-200 mesh) with EtOAc-petroleum ether $\left(60-90^{\circ} \mathrm{C}\right)$ as the eluent. Solutions were concentrated at $<60^{\circ} \mathrm{C}$ under reduced pressure.

Allyl 2-O-acetyl-3,4,6-tri-O-benzoyl- $\alpha$-D-mannopyranosyl- $(1 \rightarrow 2)$ - 3,4,6-tri-O-benzoyl- $\alpha$-D-mannopyranosyl- $(1 \rightarrow 3)-4,6$ - O-benzylidene - $\alpha-\mathrm{D}$ - mannopyranoside (7). - The disaccharide donor $\mathbf{5}^{10}(1153 \mathrm{mg}, 1 \mathrm{mmol})$ and the monosaccharide acceptor 6 (308 $\mathrm{mg}, 1 \mathrm{mmol})$ were dried together under high vacuum for $2 \mathrm{~h}$, then dissolved in anhyd $\mathrm{CH}_{2} \mathrm{Cl}_{2}(50 \mathrm{~mL})$. TMSOTf $(15 \mu \mathrm{L}$, 0.08 equiv) was added dropwise at $-20^{\circ} \mathrm{C}$ with $\mathrm{N}_{2}$ protection. The reaction mixture was stirred for $2 \mathrm{~h}$, during which time the reaction temperature was gradually raised to ambient temperature. Then the mixture was neutralized with triethylamine and concentrated under reduced pressure to an oily residue. Purification by column chromatography (1:1.5 petroleum etherEtOAc) gave 7 (1194 mg, 92\%) as colorless crystals: mp $135-137{ }^{\circ} \mathrm{C} ;[\alpha]_{\mathrm{D}}-2.0^{\circ}$ (c $\left.0.9, \mathrm{CHCl}_{3}\right) ;{ }^{1} \mathrm{H}$ NMR $\left(\mathrm{CDCl}_{3}\right): \delta 8.06-7.32(\mathrm{~m}, 30 \mathrm{H}, \mathrm{Bz}-\mathrm{H}), 5.95-5.85(\mathrm{~m}, 3$ $\left.\mathrm{H}, \mathrm{H}-3^{\prime \prime}, \mathrm{H}-4^{\prime \prime}, \mathrm{CH}_{2}=\mathrm{CH}-\mathrm{CH}_{2}\right), 5.83\left(\mathrm{dd}, J_{2,3} 3.0, J_{3,4}\right.$ $\left.10.0 \mathrm{~Hz}, 1 \mathrm{H}, \mathrm{H}-3^{\prime}\right), 5.77\left(\mathrm{dd}, J_{4,5} 10.1 \mathrm{~Hz}, 1 \mathrm{H}, \mathrm{H}-4^{\prime}\right)$, $5.61\left(\mathrm{dd}, J_{2,3} 3.0, J_{1,2} 1.5 \mathrm{~Hz}, 1 \mathrm{H}, \mathrm{H}-2^{\prime}\right), 5.56(\mathrm{~s}, 1 \mathrm{H}$,
$\mathrm{Ph}-\mathrm{CH}), 5.40\left(\mathrm{dd}, 1 \mathrm{H}, \mathrm{CH}_{2}=\mathrm{CH}-\mathrm{CH}_{2}\right), 5.30(\mathrm{dd}, 1 \mathrm{H}$, $\left.\mathrm{CH}_{2}=\mathrm{CH}-\mathrm{CH}_{2}\right), 5.02\left(\mathrm{~s}, 1 \mathrm{H}, \mathrm{H}-1^{\prime \prime}\right), 4.91(\mathrm{~s}, 1 \mathrm{H}), 4.90$ $(\mathrm{s}, 1 \mathrm{H}),\left(\mathrm{H}-1, \mathrm{H}-1^{\prime}\right), 4.60-4.50(\mathrm{~m}, 2 \mathrm{H}), 4.47$ (dd, $J_{2,3}$ 2.8, $\left.J_{1,2} 1.3 \mathrm{~Hz}, 1 \mathrm{H}, \mathrm{H}-2\right), 4.36$ (dd, $J_{3,4} 10.0 \mathrm{~Hz}, 1 \mathrm{H}$, $\mathrm{H}-3)$, 4.29-4.17 (m, 3 H), 4.15-4.05 (m, 3 H), 4.02$3.95(\mathrm{~m}, 3 \mathrm{H}), 3.88\left(\mathrm{dd}, 1 \mathrm{H}, \mathrm{CH}_{2}=\mathrm{CH}-\mathrm{CH}_{2}\right), 3.82(\mathrm{dd}$, $\left.J_{4,5} 10.0 \mathrm{~Hz}, 1 \mathrm{H}, \mathrm{H}-4\right), 2.01\left(\mathrm{~s}, 3 \mathrm{H}, \mathrm{CH}_{3} \mathrm{CO}\right) ;{ }^{13} \mathrm{C}$ NMR $\left(\mathrm{CDCl}_{3}\right): \delta 169.29,\left(\mathrm{CH}_{3} \mathrm{CO}\right), 166.48,165.89$, $165.69,165.67,165.45,165.07,\left(4 \mathrm{C}_{6} \mathrm{H}_{5} C \mathrm{O}\right), 101.92$, $(\mathrm{Ph}-\mathrm{C}), 99.95,99.39,98.87,\left(\mathrm{C}-1^{\mathrm{I}-\mathrm{III}}\right), 78.66$, (C-3), 75.84, 73.32, 71.28, 70.51, 69.83, 69.46, 69.44, 68.91, $67.89,66.85,64.14,63.40,62.77,55.01\left(\mathrm{C}-2,3,4,5,6^{\mathrm{I}-\mathrm{III}}\right)$. Anal. Calcd for $\mathrm{C}_{72} \mathrm{H}_{66} \mathrm{O}_{23}: \mathrm{C}, 66.56 ; \mathrm{H}, 5.08$. Found: C, 66.68; H, 5.01.

Allyl 2-O-acetyl-3,4,6-tri-O-benzoyl- $\alpha$-D-mannopyranosyl-( $1 \rightarrow 2)$ - 3,4,6-tri-O-benzoyl- $\alpha$-D-mannopyranosyl-(1 $\rightarrow 3)-2-\mathrm{O}$-acetyl-4,6-O-benzylidene- $\alpha$-D-mannopyranoside $(\mathbf{8})$. - Acetic anhydride $(1 \mathrm{~mL}, 10 \mathrm{mmol})$ was added dropwise to a solution of $7(1298 \mathrm{mg}, 1$ $\mathrm{mmol})$ in pyridine $(30 \mathrm{~mL})$, and the mixture was stirred overnight at rt. TLC (1.5:1 petroleum ether-EtOAc) indicated that the reaction was complete. The mixture was diluted with $\mathrm{CH}_{2} \mathrm{Cl}_{2}$, washed with $1 \mathrm{~N} \mathrm{HCl}$, water, and satd aq $\mathrm{NaHCO}_{3}$. The organic layers were combined, dried, and concentrated. Purification by column 
chromatography (2:1 petroleum ether-EtOAc) quantitatively gave $\mathbf{8}$ as colorless crystals: $\mathrm{mp} 130-132{ }^{\circ} \mathrm{C}$; $[\alpha]_{\mathrm{D}}-7.3^{\circ}\left(c 0.9, \mathrm{CHCl}_{3}\right) ;{ }^{1} \mathrm{H}$ NMR $\left(\mathrm{CDCl}_{3}\right): \delta 8.09-$ $7.35(\mathrm{~m}, 30 \mathrm{H}, \mathrm{Bz}-\mathrm{H}), 5.97\left(\mathrm{t}, J_{3,4}=J_{4,5} 10.0 \mathrm{~Hz}, 1 \mathrm{H}\right.$, $\left.\mathrm{H}-4^{\prime \prime}\right)$, 5.93-5.85 (m, $\left.2 \mathrm{H}, \mathrm{H}-3^{\prime \prime}, \mathrm{CH}_{2}=\mathrm{CH}-\mathrm{CH}_{2}\right), 5.81$ $\left(\mathrm{dd}, J_{2,3} 3.0, J_{3,4} 10.0 \mathrm{~Hz}, 1 \mathrm{H}, \mathrm{H}-3^{\prime}\right), 5.72\left(\mathrm{t}, J_{4,5} 10.1\right.$ $\left.\mathrm{Hz}, 1 \mathrm{H}, \mathrm{H}-4^{\prime}\right), 5.63\left(\mathrm{dd}, J_{2,3} 3.0, J_{1,2} 1.5 \mathrm{~Hz}, 1 \mathrm{H}\right.$, $\left.\mathrm{H}-2^{\prime \prime}\right), 5.55(\mathrm{~s}, 1 \mathrm{H}, \mathrm{Ph}-\mathrm{CH}), 5.42\left(\mathrm{dd}, J_{2,3} 2.8, J_{1,2} 1.4\right.$ $\mathrm{Hz}, 1 \mathrm{H}, \mathrm{H}-2), 5.32\left(\mathrm{dd}, 1 \mathrm{H}, \mathrm{CH}_{2}=\mathrm{CH}-\mathrm{CH}_{2}\right), 5.24(\mathrm{dd}$, $\left.1 \mathrm{H}, \mathrm{CH}_{2}=\mathrm{CH}-\mathrm{CH}_{2}\right), 5.05\left(\mathrm{~d}, 1 \mathrm{H}, \mathrm{H}-1^{\prime \prime}\right), 4.96(\mathrm{~s}, 1 \mathrm{H})$, $4.86(\mathrm{~s}, 1 \mathrm{H}),\left(\mathrm{H}-1, \mathrm{H}-1^{\prime}\right), 4.60-4.50(\mathrm{~m}, 2 \mathrm{H}), 4.40(\mathrm{dd}$, $\left.J_{2,3} 2.8, J_{1,2} 1.3 \mathrm{~Hz}, 1 \mathrm{H}, \mathrm{H}-2\right), 4.36\left(\mathrm{dd}, J_{3,4} 10.0 \mathrm{~Hz}, 1\right.$ $\mathrm{H}, \mathrm{H}-3), 4.29-4.20(\mathrm{~m}, 2 \mathrm{H}), 4.15-4.05(\mathrm{~m}, 3 \mathrm{H})$, $4.02-3.95(\mathrm{~m}, 3 \mathrm{H}), 3.88\left(\mathrm{dd}, 1 \mathrm{H}, \mathrm{CH}_{2}=\mathrm{CH}-\mathrm{CH}_{2}\right), 3.84$ $\left(\mathrm{t}, J_{4,5} 10.0 \mathrm{~Hz}, 1 \mathrm{H}, \mathrm{H}-4\right), 2.39\left(\mathrm{~s}, 3 \mathrm{H}, \mathrm{CH}_{3} \mathrm{CO}\right) 1.97$ (s, $3 \mathrm{H}, \mathrm{CH}_{3} \mathrm{CO}$ ). Anal. Calcd for $\mathrm{C}_{74} \mathrm{H}_{68} \mathrm{O}_{24}: \mathrm{C}, 66.27$; H, 5.07. Found: C, 66.40; H, 5.00.

Allyl 2-O-acetyl-3,4,6-tri-O-benzoyl- $\alpha$-D-mannopyranosyl- $(1 \rightarrow 2)$ - 3,4,6-tri-O-benzoyl- $\alpha$-D-mannopyranosyl-(1 $\rightarrow 3)$-2-O-acetyl- $\alpha$-D-mannopyranoside

(9).-

Acetyl chloride $(0.05 \mathrm{~mL}, 0.7 \mathrm{mmol})$ was added dropwise to a solution of 8 (1340 mg, $1 \mathrm{mmol})$ in $\mathrm{MeOH}(50$ $\mathrm{mL}$ ), and the mixture was stirred overnight at rt. TLC (1.5:1 petroleum ether-EtOAc) indicated that the reaction was complete, and the mixture was concentrated. Purification by column chromatography (1.5:1 petroleum ether-EtOAc) gave 9 (1127 $\mathrm{mmg}, 90 \%)$ as a colorless solid: $[\alpha]_{\mathrm{D}}-10.3^{\circ}\left(c\right.$ 1.3, $\left.\mathrm{CHCl}_{3}\right) ;{ }^{1} \mathrm{H}$ NMR $\left(\mathrm{CDCl}_{3}\right): \delta 8.06-7.26(\mathrm{~m}, 30 \mathrm{H}, \mathrm{Bz}-\mathrm{H}), 5.96(\mathrm{dd}$, $\left.J_{3,4}=J_{4,5} 10.0 \mathrm{~Hz}, 1 \mathrm{H}, \mathrm{H}-4^{\prime \prime}\right), 5.90-5.78\left(\mathrm{~m}, 3 \mathrm{H}, \mathrm{H}-4^{\prime}\right.$, $\left.\mathrm{H}-3^{\prime \prime}, \mathrm{CH}_{2}=\mathrm{CH}-\mathrm{CH}_{2}\right), 5.75$ (dd, $J_{2,3} 3.0, J_{3,4} 10.0 \mathrm{~Hz}, 1$ $\left.\mathrm{H}, \mathrm{H}-3^{\prime}\right), 5.65\left(\mathrm{dd}, J_{2,3} 2.9, J_{1,2} 1.4 \mathrm{~Hz}, 1 \mathrm{H}, \mathrm{H}-2^{\prime \prime}\right), 5.62$ (dd, $\left.J_{2,3} 2.8, J_{1,2} 1.4 \mathrm{~Hz}, 1 \mathrm{H}, \mathrm{H}-2\right), 5.38\left(\mathrm{~s}, 1 \mathrm{H}, \mathrm{H}-1^{\prime \prime}\right)$, $5.28\left(\mathrm{dd}, 1 \mathrm{H}, \quad \mathrm{CH}_{2}=\mathrm{CH}-\mathrm{CH}_{2}\right), 5.20(\mathrm{dd}, 1 \mathrm{H}$, $\left.\mathrm{CH}_{2}=\mathrm{CH}-\mathrm{CH}_{2}\right), 5.12\left(\mathrm{~s}, 1 \mathrm{H}, \mathrm{H}-1^{\prime}\right), 4.84(\mathrm{~s}, 1 \mathrm{H}, \mathrm{H}-1)$, $4.64-4.38(\mathrm{~m}, 8 \mathrm{H}), 4.18(\mathrm{~m}, 1 \mathrm{H}), 4.14\left(\mathrm{dd}, J_{2,3} 3.0, J_{3,4}\right.$ $10.0 \mathrm{~Hz}, 1 \mathrm{H}, \mathrm{H}-3), 4.06\left(\mathrm{dd}, J_{3,4}=J_{4,5} 10.0 \mathrm{~Hz}, 1 \mathrm{H}\right.$, $\mathrm{H}-4), 3.95\left(\mathrm{dd}, 1 \mathrm{H}, \mathrm{CH}_{2}=\mathrm{CH}-\mathrm{CH}_{2}\right), 3.78\left(\mathrm{dd}, J_{2,3} 2.9\right.$, $\left.J_{1,2} 1.4 \mathrm{~Hz}, 1 \mathrm{H}, \mathrm{H}-2^{\prime}\right), 3.70\left(\mathrm{dd}, 1 \mathrm{H}, \mathrm{CH}_{2}=\mathrm{CH}-\mathrm{CH}_{2}\right.$ ), $2.22\left(\mathrm{~s}, 3 \mathrm{H}, \mathrm{CH}_{3} \mathrm{CO}\right) 2.02\left(\mathrm{~s}, 3 \mathrm{H}, \mathrm{CH}_{3} \mathrm{CO}\right) ;{ }^{13} \mathrm{C} \mathrm{NMR}$ $\left(\mathrm{CDCl}_{3}\right): \delta 170.18,169.84,\left(2 \mathrm{CH}_{3} \mathrm{CO}\right), 166.28,166.09$, $165.49,165.47,164.44,164.42,\left(6 \mathrm{C}_{6} \mathrm{H}_{5} \mathrm{CO}\right), 99.97$, 99.69, 97.97, (C-1 $\left.{ }^{\mathrm{I}-\mathrm{III}}\right), 78.60,(\mathrm{C}-3), 75.64,72.37,71.20$, $70.83,69.54,69.48,69.10,68.97,68.44,68.07,67.17$,

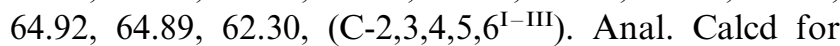
$\mathrm{C}_{67} \mathrm{H}_{64} \mathrm{O}_{24}$ : C, 64.22; H, 5.11. Found: $\mathrm{C}, 63.96 ; \mathrm{H}, 5.14$.

2-O-Acetyl-3,4,6-tri-O-benzoyl- $\alpha$-D-mannopyranosyl$(1 \rightarrow 2)-3,4,6$-tri-O-benzoyl- $\alpha$-D-mannopyranosyl- $(1 \rightarrow 3)$ [-2-O-acetyl-3,4,6-tri-O-benzoyl- $\alpha$-D-mannopyranosyl$(1 \rightarrow 2)-3,4,6$-tri-O-benzoyl- $\alpha$-D-mannopyranosyl- $(1 \rightarrow$ 6)]-1,2-O-ethylidene- $\beta$-D-mannopyranose (11). - The disaccharide donor $5(1268 \mathrm{mg}, 1.1 \mathrm{mmol})$ and the monosaccharide acceptor $10(103 \mathrm{mg}, 0.5 \mathrm{mmol})$ were dried together under high vacuum for $2 \mathrm{~h}$, then dissolved in anhyd $\mathrm{CH}_{2} \mathrm{Cl}_{2}(30 \mathrm{~mL})$. TMSOTf $(15 \mu \mathrm{L}, 0.08$ equiv) was added dropwise at $-20{ }^{\circ} \mathrm{C}$ with $\mathrm{N}_{2}$ protec- tion. The reaction mixture was stirred for $3 \mathrm{~h}$, during which time the reaction temperature was gradually raised to ambient temperature. Then the mixture was neutralized with triethylamine and concentrated under reduced pressure to give an oily residue. Purification by column chromatography $(1: 1.5$ petroleum etherEtOAc) gave $11(940 \mathrm{mg}, 86 \%)$ as a colorless solid: $\mathrm{mp}$ $141-144{ }^{\circ} \mathrm{C} ;[\alpha]_{\mathrm{D}}-11.5^{\circ}$ (c 1.1, $\left.\mathrm{CHCl}_{3}\right) ;{ }^{1} \mathrm{H}$ NMR $\left(\mathrm{CDCl}_{3}\right): \delta 7.98-7.25(\mathrm{~m}, 60 \mathrm{H}, \mathrm{Bz}-\mathrm{H}), 6.03-5.85(\mathrm{~m}, 8$ $\mathrm{H}), 5.67(\mathrm{dd}, J 3.0, J 1.7 \mathrm{~Hz}, 1 \mathrm{H}), 5.64(\mathrm{dd}, J 3.1, J 1.7$ $\mathrm{Hz}, 1 \mathrm{H}), 5.53$ (s, $1 \mathrm{H}), 5.26\left(\mathrm{q}, 1 \mathrm{H}, \mathrm{CH}_{3}-\mathrm{CH}\right), 5.20$ (s, $1 \mathrm{H}), 5.12(\mathrm{~d}, J 1.6 \mathrm{~Hz}, 1 \mathrm{H}), 5.08(\mathrm{~d}, J 1.7 \mathrm{~Hz}, 1 \mathrm{H})$, $5.05(\mathrm{~d}, J 1.7 \mathrm{~Hz}, 1 \mathrm{H}), 4.60-4.46(\mathrm{~m}, 13 \mathrm{H}), 4.42(\mathrm{dd}$, $J 3.0, J 1.7 \mathrm{~Hz}, 1 \mathrm{H}), 4.20(\mathrm{dd}, J 3.0, J 1.7 \mathrm{~Hz}, 1 \mathrm{H})$, $4.04(\mathrm{t}, J 10.0 \mathrm{~Hz}, 1 \mathrm{H}), 3.98-3.94(\mathrm{~m}, 1 \mathrm{H}), 3.83(\mathrm{dd}$, $J 3.2, J 10.0 \mathrm{~Hz}, 1 \mathrm{H}), 3.75-3.68(\mathrm{~m}, 1 \mathrm{H}), 3.41-3.37$ $(\mathrm{m}, 1 \mathrm{H}), 2.01\left(\mathrm{~s}, 3 \mathrm{H}, \mathrm{CH}_{3} \mathrm{CO}\right), 2.00\left(\mathrm{~s}, 3 \mathrm{H}, \mathrm{CH}_{3} \mathrm{CO}\right)$, $1.50\left(\mathrm{~d}, 3 \mathrm{H}, \mathrm{CH}_{3}-\mathrm{CH}\right)$. Anal. Calcd for $\mathrm{C}_{120} \mathrm{H}_{106} \mathrm{O}_{40}$ : C, 65.87; H, 4.85. Found: C, 65.98; H, 4.80.

2-O-Acetyl-3,4,6-tri-O-benzoyl- $\alpha$-D-mannopyranosyl$(1 \rightarrow 2)-3,4,6$-tri-O-benzoyl- $\alpha$-D-mannopyranosyl- $(1 \rightarrow 3)$ [2-O-acetyl-3,4,6-tri-O-benzoyl- $\alpha$-D-mannopyranosyl$(1 \rightarrow 2)-3,4,6$-tri-O-benzoyl- $\alpha$-D-mannopyranosyl- $(1 \rightarrow$ 6)]-4-O-acetyl-1,2-O-ethylidene - $\beta$ - D-mannopyranose (12). - Acetic anhydride $(1 \mathrm{~mL}, 10 \mathrm{mmol})$ was added dropwise to a solution of $\mathbf{1 1}(2186 \mathrm{mg}, 1 \mathrm{mmol})$ in pyridine $(30 \mathrm{~mL})$, and the mixture was stirred overnight at rt. TLC (1.5:1 petroleum ether-EtOAc) indicated that the reaction was complete. The mixture was diluted with $\mathrm{CH}_{2} \mathrm{Cl}_{2}$, washed with $1 \mathrm{~N} \mathrm{HCl}$, water, and satd aq $\mathrm{NaHCO}_{3}$. The organic layers were combined, dried, and concentrated. Purification by column chromatography (2:1 petroleum ether-EtOAc) quantitatively gave 12 as a colorless solid: $\mathrm{mp} 138-139^{\circ} \mathrm{C} ;[\alpha]_{\mathrm{D}}$ $-4.5^{\circ}\left(c 1.0, \mathrm{CHCl}_{3}\right) ;{ }^{1} \mathrm{H}$ NMR $\left(\mathrm{CDCl}_{3}\right): \delta$ 8.09-7.25 $(\mathrm{m}, 60 \mathrm{H}, \mathrm{Bz}-\mathrm{H}), 6.03-5.82(\mathrm{~m}, 7 \mathrm{H}), 5.80(\mathrm{dd}, J 3.1, J$ $10.0 \mathrm{~Hz}, 1 \mathrm{H}), 5.69(\mathrm{dd}, J 3.0, J 1.7 \mathrm{~Hz}, 1 \mathrm{H}), 5.62(\mathrm{dd}$, $J$ 3.0, $J 1.7 \mathrm{~Hz}, 1 \mathrm{H}), 5.28\left(\mathrm{dd}, 1 \mathrm{H}, \mathrm{CH}_{3}-\mathrm{CH}\right), 5.22(\mathrm{~s}$, $1 \mathrm{H}), 5.20(\mathrm{t}, J 10.0 \mathrm{~Hz}, 1 \mathrm{H}), 5.18(\mathrm{~s}, 1 \mathrm{H}), 5.09(\mathrm{~s}, 1$ H), $5.08(\mathrm{~s}, 1 \mathrm{H}), 5.04(\mathrm{~s}, 1 \mathrm{H}), 4.63-4.44(\mathrm{~m}, 12 \mathrm{H})$, $4.48(\mathrm{dd}, J 3.0, J 1.7 \mathrm{~Hz}, 1 \mathrm{H}), 4.30(\mathrm{dd}, J 3.1, J 1.6 \mathrm{~Hz}$, $1 \mathrm{H}), 4.23(\mathrm{dd}, J 3.0, J 1.5 \mathrm{~Hz}, 1 \mathrm{H}), 3.90-3.80(\mathrm{~m}, 2$ $\mathrm{H}), 3.60-3.55(\mathrm{~m}, 1 \mathrm{H}), 3.46-3.40(\mathrm{~m}, 1 \mathrm{H}), 2.12(\mathrm{~s}, 3$ $\mathrm{H}, \mathrm{CH}_{3} \mathrm{CO}$ ), 2.01 (s, $\left.3 \mathrm{H}, \mathrm{CH}_{3} \mathrm{CO}\right), 2.00$ (s, $3 \mathrm{H}$, $\left.\mathrm{CH}_{3} \mathrm{CO}\right), 1.46\left(\mathrm{~d}, 3 \mathrm{H}, \mathrm{CH}_{3}-\mathrm{CH}\right)$. Anal. Calcd for $\mathrm{C}_{122} \mathrm{H}_{108} \mathrm{O}_{41}$ : C, 65.71; H, 4.85. Found: C, 65.84; $\mathrm{H}$ 4.73 .

2-O-Acetyl-3,4,6-tri-O-benzoyl- $\alpha$-D-mannopyranosyl$(1 \rightarrow 2)-3,4,6$-tri-O-benzoyl- $\alpha$-D-mannopyranosyl- $(1 \rightarrow 3)$ [2-O-acetyl-3,4,6-tri-O-benzoyl- $\alpha$-D-mannopyranosyl$(1 \rightarrow 2)-3,4,6$-tri-O-benzoyl- $\alpha$-D-mannopyranosyl- $(1 \rightarrow$ 6)]-2,4-di-O-acetyl-D-mannopyranose (13). - Compound 12 (2228 mg, $1 \mathrm{mmol}$ ) was treated with $90 \%$ $\mathrm{F}_{3} \mathrm{CCOOH}(10 \mathrm{~mL})$ at $\mathrm{rt}$ for $1 \mathrm{~h}$, and the solution was concentrated and co-concentrated with toluene. The residue was dissolved in pyridine $(10 \mathrm{~mL})$ and treated 
with $\mathrm{Ac}_{2} \mathrm{O}(3 \mathrm{~mL})$ for $2 \mathrm{~h}$. After conventional workup, the residue was subjected to column chromatography (1.5:1 petroleum ether-EtOAc) to yield 2- $O$-acetyl3,4,6-tri- $O$-benzoyl- $\alpha$-D-mannopyranosyl- $(1 \rightarrow 2)-3,4,6$ tri- $O$-benzoyl- $\alpha$-D-mannopyranosyl- $(1 \rightarrow 3)$-[2- $O$-acetyl-3,4,6-tri- $O$-benzoyl- $\alpha$-D-mannopyranosyl- $(1 \rightarrow 2)$ 3,4,6 - tri- $O$-benzoyl- $\alpha$-D-mannopyranosyl- $(1 \rightarrow 6)]-1,2,4$ tri- $O$-acetyl- $\alpha, \beta$-D-mannopyranose as a solid $(1941 \mathrm{mg}$, $85 \%)$. A solution of the solid $(1142 \mathrm{mg}, 0.5 \mathrm{mmol})$ and ammonium carbonate $(790 \mathrm{mg}, 10 \mathrm{mmol})$ in DMF (20 $\mathrm{mL}$ ) was stirred for $12 \mathrm{~h}$ at $\mathrm{rt}$, at the end of which time TLC (2:1 petroleum ether-EtOAc) indicated that the reaction was complete. Water was added, and the mixture was diluted with $\mathrm{CH}_{2} \mathrm{Cl}_{2}$, washed with $1 \mathrm{~N} \mathrm{HCl}$, water, and satd aq $\mathrm{NaHCO}_{3}$. The organic layer was combined, dried, and concentrated. The residue thus obtained was passed through a short silica gel column with 2:1 petroleum ether-EtOAc as the eluent to give 13 as a solid consisting predominantly of the $\alpha$ anomer (1021 mg, 91\%): $[\alpha]_{\mathrm{D}}-13.2^{\circ}\left(c 1.1, \mathrm{CHCl}_{3}\right) ;{ }^{1} \mathrm{H}$ NMR $\left(\mathrm{CDCl}_{3}\right): \delta 8.01-7.26(\mathrm{~m}, 60 \mathrm{H}, \mathrm{Bz}-\mathrm{H}), 5.95-5.83(\mathrm{~m}, 7$ H), $5.70(\mathrm{dd}, J 3.0, J 10.0 \mathrm{~Hz}, 1 \mathrm{H}), 5.64(\mathrm{dd}, J 3.1, J$ $1.5 \mathrm{~Hz}, 1 \mathrm{H}), 5.60(\mathrm{dd}, J 3.0, J 1.5 \mathrm{~Hz}, 1 \mathrm{H}), 5.49(\mathrm{dd}$, $J 3.0, J 1.5 \mathrm{~Hz}, 1 \mathrm{H}), 5.40(\mathrm{~s}, 1 \mathrm{H}), 5.24(\mathrm{~s}, 1 \mathrm{H}), 5.22$ $(\mathrm{t}, J 10.0 \mathrm{~Hz}, 1 \mathrm{H}), 5.20(\mathrm{~s}, 1 \mathrm{H}), 5.08(\mathrm{~s}, 1 \mathrm{H}), 5.02(\mathrm{~s}$, $1 \mathrm{H}), 4.66-4.37(\mathrm{~m}, 14 \mathrm{H}), 4.22(\mathrm{dd}, J 3.0, J 1.5 \mathrm{~Hz}, 1$ $\mathrm{H}), 4.00-3.95(\mathrm{~m}, 1 \mathrm{H}), 3.77-3.73(\mathrm{dd}, 1 \mathrm{H}), 3.58-3.55$ $(\mathrm{m}, 1 \mathrm{H}), 2.30\left(\mathrm{~s}, 3 \mathrm{H}, \mathrm{CH}_{3} \mathrm{CO}\right), 2.19\left(\mathrm{~s}, 3 \mathrm{H}, \mathrm{CH}_{3} \mathrm{CO}\right)$, $2.01\left(\mathrm{~s}, 3 \mathrm{H}, \mathrm{CH}_{3} \mathrm{CO}\right), 2.00$ (s, $\left.3 \mathrm{H}, \mathrm{CH}_{3} \mathrm{CO}\right) ;{ }^{13} \mathrm{C} \mathrm{NMR}$ $\left(\mathrm{CDCl}_{3}\right): \delta 170.52,170.04,169.31,169.26,\left(4 \mathrm{CH}_{3} \mathrm{CO}\right)$, $166.66,166.53,166.45,166.15,165.70,165.67,165.65$, $165.55,165.42,165.28,165.11,164.98,\left(12 \mathrm{C}_{6} \mathrm{H}_{5} C \mathrm{O}\right)$, 100.47, 99.76, 99.36, 98.97, $92.36\left(\mathrm{C}-1^{\mathrm{I}-\mathrm{V}}\right), 79.08(\mathrm{C}-3)$, 77.28, 77.28 (C-2), 72.86, 71.32, 70.59, 70.06, 69.94, $69.73,69.70,69.67,69.64,69.61,69.58,69.38,69.11$, 68.47, 68.20, 67.49, 67.12, 66.88, 63.97, 63.86, 63.33, 63.04, (C-2,3,4,5,6 $\left.{ }^{\mathrm{I}-\mathrm{V}}\right)$. Anal. Calcd for $\mathrm{C}_{122} \mathrm{H}_{108} \mathrm{O}_{42}$ : C, 65.24; H, 4.81. Found: C, 65.08; H, 4.88.

2-O-Acetyl-3,4,6-tri-O-benzoyl- $\alpha$-D-mannopyranosyl$(1 \rightarrow 2)-3,4,6$-tri-O-benzoyl- $\alpha$-D-mannopyranosyl- $(1 \rightarrow 3)$ [2-O-acetyl-3,4,6-tri-O-benzoyl- $\alpha$-D-mannopyranosyl$(1 \rightarrow 2)-3,4,6$-tri-O-benzoyl- $\alpha$-D-mannopyranosyl- $(1 \rightarrow$ 6)]-2,4-di-O-acetyl- $\alpha$-D-mannopyranosyl trichloroacetimidate (14). - The compound 13 (2244 mg, $1 \mathrm{mmol})$ was dissolved in $\mathrm{CH}_{2} \mathrm{Cl}_{2}(20 \mathrm{~mL})$, and $\mathrm{CCl}_{3} \mathrm{CN}(0.1$ $\mathrm{mL}, 1 \mathrm{mmol})$ and $\mathrm{DBU}(14 \mu \mathrm{L}, 0.1 \mathrm{mmol})$ were added. The reaction mixture was stirred for $2 \mathrm{~h}$, at the end of which time TLC (2:1 petroleum ether-EtOAc) indicated that the reaction was complete. Concentration of the reaction mixture, followed by purification on a silica gel column with 2:1 petroleum ether-EtOAc as eluent furnished the pentasaccharide donor $\mathbf{1 4}$ as crystals in a good yield $(2150 \mathrm{mg}, 90 \%): \mathrm{mp} 135-137^{\circ} \mathrm{C}$; $[\alpha]_{\mathrm{D}}-9.5^{\circ}\left(c 1.3, \mathrm{CHCl}_{3}\right) ;{ }^{1} \mathrm{H}$ NMR $\left(\mathrm{CDCl}_{3}\right): \delta 9.03$ $(\mathrm{s}, 1 \mathrm{H}, \mathrm{C}=\mathrm{NH}), 8.10-7.26(\mathrm{~m}, 60 \mathrm{H}, \mathrm{Bz}-\mathrm{H}), 6.35(\mathrm{~d}$, $\left.J_{1,2} 1.5 \mathrm{~Hz}, 1 \mathrm{H}, \mathrm{H}-1\right), 6.05(\mathrm{t}, J 10.0 \mathrm{~Hz}, 1 \mathrm{H}), 5.96(\mathrm{t}$,
$J 10.1 \mathrm{~Hz}, 2 \mathrm{H}), 5.91(\mathrm{t}, J 10.1 \mathrm{~Hz}, 1 \mathrm{H}), 5.88(\mathrm{dd}, J 3.0$, $J 10.0 \mathrm{~Hz}, 1 \mathrm{H}), 5.85(\mathrm{dd}, J 3.0, J 10.0 \mathrm{~Hz}, 1 \mathrm{H}), 5.80$ $(\mathrm{dd}, J 3.0, J 10.1 \mathrm{~Hz}, 1 \mathrm{H}), 5.74(\mathrm{dd}, J 3.0, J 10.1 \mathrm{~Hz}$, $1 \mathrm{H}), 5.68-5.66(\mathrm{~m}, 2 \mathrm{H}), 5.64(\mathrm{dd}, J 3.0, J 1.5 \mathrm{~Hz}, 1$ $\mathrm{H}), 5.45(\mathrm{t}, J 10.0 \mathrm{~Hz}, 1 \mathrm{H}), 5.43(\mathrm{~s}, 1 \mathrm{H}), 5.17(\mathrm{~s}, 1 \mathrm{H})$, $5.09(\mathrm{~s}, 2 \mathrm{H}), 4.61-4.43(\mathrm{~m}, 13 \mathrm{H}), 4.33(\mathrm{dd}, J 3.0, J 1.5$ $\mathrm{Hz}, 1 \mathrm{H}), 4.30(\mathrm{dd}, J 3.0, J 1.5 \mathrm{~Hz}, 1 \mathrm{H}), 4.22-4.18(\mathrm{~m}$, $1 \mathrm{H}), 3.89-3.84(\mathrm{~m}, 1 \mathrm{H}), 3.59-3.56(\mathrm{~m}, 1 \mathrm{H}), 2.26(\mathrm{~s}$, $3 \mathrm{H}, \mathrm{CH}_{3} \mathrm{CO}$ ), $2.10\left(\mathrm{~s}, 3 \mathrm{H}, \mathrm{CH}_{3} \mathrm{CO}\right), 2.02(\mathrm{~s}, 3 \mathrm{H}$, $\mathrm{CH}_{3} \mathrm{CO}$ ), $2.00\left(\mathrm{~s}, 3 \mathrm{H}, \mathrm{CH}_{3} \mathrm{CO}\right.$ ). Anal. Calcd for $\mathrm{C}_{124} \mathrm{H}_{108} \mathrm{Cl}_{3} \mathrm{NO}_{42}$ : C, 62.30; H, 4.52. Found: C, 62.45; $\mathrm{H}, 4.40$.

Allyl 2-O-acetyl-3,4,6-tri-O-benzoyl- $\alpha$-D-mannopyranosyl-( $1 \rightarrow 2)-3,4,6$-tri-O-benzoyl- $\alpha$-D-mannopyranosyl - (1 $\rightarrow 3)$ - $\{2$ - O - acetyl - 3,4,6 - tri - O - benzoyl - $\alpha$ - Dmannopyranosyl - $(1 \rightarrow 2)$ - 3,4,6 - tri - O - benzoyl - $\alpha$ - Dmannopyranosyl- $(1 \rightarrow 3)$-[2-O-acetyl-3,4,6-tri-O-benzo$y l-\alpha$-D-mannopyranosyl- $(1 \rightarrow 2)-3,4,6$ - tri-O-benzoyl- $\alpha$ $\mathrm{D}$ - mannopyranosyl - $(1 \rightarrow 6)]-2,4-$ di - O - acetyl - $\alpha$ - Dmannopyranosyl- $(1 \rightarrow 6)\}-2-\mathrm{O}$-acetyl- $\alpha$-D-mannopyranoside (15). - The pentasaccharide donor 14 (1193 mg, $0.5 \mathrm{mmol})$ and the trisaccharide acceptor 9 (626 mg, 0.5 mmol) were dried together under high vacuum for $2 \mathrm{~h}$, then dissolved in anhyd $\mathrm{CH}_{2} \mathrm{Cl}_{2}(30 \mathrm{~mL})$. TMSOTf (7.5 $\mu \mathrm{L}, 0.08$ equiv) was added dropwise at $-20^{\circ} \mathrm{C}$ with $\mathrm{N}_{2}$ protection. The reaction mixture was stirred for $3 \mathrm{~h}$, during which time the reaction temperature was gradually raised to ambient temperature. Then the mixture was neutralized with triethylamine and concentrated under reduced pressure to an oily residue. Purification by column chromatography $(1: 1$ petroleum etherEtOAc) gave $15(1426 \mathrm{mg}, 82 \%)$ as a colorless solid: $[\alpha]_{\mathrm{D}}-18.0^{\circ}\left(c \quad 0.9, \mathrm{CHCl}_{3}\right) ;{ }^{1} \mathrm{H}$ NMR $\left(\mathrm{CDCl}_{3}\right): \delta$ $8.02-7.26(\mathrm{~m}, 90 \mathrm{H}, \mathrm{Bz}-\mathrm{H}), 6.29(\mathrm{t}, J 9.9 \mathrm{~Hz}, 1 \mathrm{H}), 6.03$ $(\mathrm{t}, J 9.9 \mathrm{~Hz}, 1 \mathrm{H}), 5.99-5.84(\mathrm{~m}, 6 \mathrm{H}), 5.78-5.70(\mathrm{~m}, 3$ $\mathrm{H}), 5.67-5.60(\mathrm{~m}, 3 \mathrm{H}), 5.56(\mathrm{dd}, J 3.0, J 1.5 \mathrm{~Hz}, 1 \mathrm{H})$, $5.48-5.30(\mathrm{~m}, 6 \mathrm{H}), 5.25(\mathrm{~s}, 1 \mathrm{H}), 5.23(\mathrm{~s}, 1 \mathrm{H}), 5.15(\mathrm{~s}$, $1 \mathrm{H}), 5.12(\mathrm{~s}, 1 \mathrm{H}), 5.08(\mathrm{~s}, 1 \mathrm{H}), 5.04(\mathrm{~s}, 1 \mathrm{H}), 4.94(\mathrm{~s}$, $1 \mathrm{H}), 4.88(\mathrm{~s}, 1 \mathrm{H}), 4.70-4.40(\mathrm{~m}, 23 \mathrm{H}), 4.22(\mathrm{dd}, J 3.0$, $J 1.5 \mathrm{~Hz}, 1 \mathrm{H}), 4.20(\mathrm{dd}, J 3.0, J 9.9 \mathrm{~Hz}, 1 \mathrm{H})$, $3.87-3.76(\mathrm{~m}, 3 \mathrm{H}), 3.70(\mathrm{t}, J 9.9 \mathrm{~Hz}, 1 \mathrm{H}), 3.60-3.45$ $(\mathrm{m}, 3 \mathrm{H}), 2.29\left(\mathrm{~s}, 3 \mathrm{H}, \mathrm{CH}_{3} \mathrm{CO}\right), 2.20\left(\mathrm{~s}, 3 \mathrm{H}, \mathrm{CH}_{3} \mathrm{CO}\right)$, 2.08 (s, $3 \mathrm{H}, \mathrm{CH}_{3} \mathrm{CO}$ ), 2.01 (s, $3 \mathrm{H}, 2 \mathrm{CH}_{3} \mathrm{CO}$ ), 2.09 (s, $3 \mathrm{H}, \mathrm{CH}_{3} \mathrm{CO}$ ), 1.95 (s, $6 \mathrm{H}, 2 \mathrm{CH}_{3} \mathrm{CO}$ ); MALDI-TOF MS: Calcd for $\mathrm{C}_{189} \mathrm{H}_{170} \mathrm{O}_{65}: 3478.99$ [M]. Found: 3479 [M]. Anal. Calcd for $\mathrm{C}_{189} \mathrm{H}_{170} \mathrm{O}_{65}$ : C, 65.21; H, 4.89. Found: C, 65.36; H, 4.76 .

Allyl 2-O-acetyl-3,4,6-tri-O-benzoyl- $\alpha$-D-mannopyranosyl-( $1 \rightarrow 2)$ - 3,4,6-tri-O-benzoyl- $\alpha$-D-mannopyranosyl- $(1 \rightarrow 3)$ - $\{2-\mathrm{O}$ - acetyl - 3,4,6- tri - O - benzoyl - $\alpha$ - Dmannopyranosyl - $(1 \rightarrow 2)$ - 3,4,6 - tri - O - benzoyl - $\alpha$ - Dmannopyranosyl-(1 $\rightarrow 3)$-[2-O-acetyl-3,4,6-tri-O-benzo$y l$ - $\alpha$-D-mannopyranosyl- $(1 \rightarrow 2)-3,4,6$-tri-O-benzoyl- $\alpha$ D-mannopyranosyl- $(1 \rightarrow 6)]-2,4-d i$-O-acetyl- $\alpha$-D-mannopyranosyl- $(1 \rightarrow 6)\}-2,4$-di-O-acetyl- $\alpha$ - D-mannopyranoside (16).-Acetic anhydride $(1 \mathrm{~mL}, 10 \mathrm{mmol})$ was added dropwise to a solution of 15 (1043 $\mathrm{mg}, 0.3 \mathrm{mmol})$ 
in pyridine $(20 \mathrm{~mL})$, and the mixture was stirred overnight at rt. TLC (1.5:1 petroleum ether-EtOAc) indicated that the reaction was complete. The mixture was diluted with $\mathrm{CH}_{2} \mathrm{Cl}_{2}$, washed with $1 \mathrm{~N} \mathrm{HCl}$, water, and satd aq $\mathrm{NaHCO}_{3}$. The organic layers were combined, dried, and concentrated. Purification by column chromatography (1.5:1 petroleum ether-EtOAc) gave 16 quantitatively as a colorless solid: $[\alpha]_{\mathrm{D}}-14.5^{\circ}(c$ 1.0, $\left.\mathrm{CHCl}_{3}\right) ;{ }^{1} \mathrm{H}$ NMR $\left(\mathrm{CDCl}_{3}\right): \delta 8.02-7.26(\mathrm{~m}, 90 \mathrm{H}$, $\mathrm{Bz}-\mathrm{H}), 6.31(\mathrm{t}, J 9.9 \mathrm{~Hz}, 1 \mathrm{H}), 6.01(\mathrm{t}, J 9.9 \mathrm{~Hz}, 1 \mathrm{H})$, $5.98-5.83(\mathrm{~m}, 7 \mathrm{H}), 5.78-5.70(\mathrm{~m}, 3 \mathrm{H}), 5.67-5.60(\mathrm{~m}$, $3 \mathrm{H}), 5.56(\mathrm{dd}, J 3.0, J 1.5 \mathrm{~Hz}, 1 \mathrm{H}), 5.48(\mathrm{t}, J 9.9 \mathrm{~Hz}$, $1 \mathrm{H}), 5.45-5.30(\mathrm{~m}, 5 \mathrm{H}), 5.23(\mathrm{~s}, 1 \mathrm{H}), 5.20(\mathrm{~s}, 1 \mathrm{H})$, $5.12(\mathrm{~s}, 1 \mathrm{H}), 5.10(\mathrm{~s}, 1 \mathrm{H}), 5.08(\mathrm{~s}, 1 \mathrm{H}), 5.03(\mathrm{~s}, 1 \mathrm{H})$, $4.95(\mathrm{~s}, 1 \mathrm{H}), 4.92(\mathrm{~s}, 1 \mathrm{H}), 4.70-4.42(\mathrm{~m}, 23 \mathrm{H}), 4.25$ $(\mathrm{dd}, J 3.0, J 1.5 \mathrm{~Hz}, 1 \mathrm{H}), 4.20(\mathrm{dd}, J 3.0, J 9.9 \mathrm{~Hz}, 1$ $\mathrm{H}), 3.87-3.76(\mathrm{~m}, 2 \mathrm{H}), 3.74(\mathrm{t}, J 9.9 \mathrm{~Hz}, 1 \mathrm{H})$, $3.60-3.45(\mathrm{~m}, 3 \mathrm{H}), 2.28\left(\mathrm{~s}, 3 \mathrm{H}, \mathrm{CH}_{3} \mathrm{CO}\right), 2.23(\mathrm{~s}, 3 \mathrm{H}$, $\left.\mathrm{CH}_{3} \mathrm{CO}\right), 2.08\left(\mathrm{~s}, 3 \mathrm{H}, \mathrm{CH}_{3} \mathrm{CO}\right), 2.01$ (s, $3 \mathrm{H}, 2$ $\mathrm{CH}_{3} \mathrm{CO}$ ), 2.00 (s, $3 \mathrm{H}, \mathrm{CH}_{3} \mathrm{CO}$ ), 2.09 (s, $3 \mathrm{H}, \mathrm{CH}_{3} \mathrm{CO}$ ), $1.96\left(\mathrm{~s}, 3 \mathrm{H}, \mathrm{CH}_{3} \mathrm{CO}\right) ;{ }^{13} \mathrm{C} \mathrm{NMR}\left(\mathrm{CDCl}_{3}\right): \delta 170.11$, $170.08,169.56,169.31,169.82,169.79,169.76$, (7 $\left.\mathrm{CH}_{3} \mathrm{CO}\right), 165.92,165.89,165.83,165.75,165.56,165.51$, $165.28,165.20,165.01,165.05,164.99,164.95,164.89$, $164.68,164.56,164.52,164.48,164.27,\left(18 \mathrm{C}_{6} \mathrm{H}_{5} C \mathrm{O}\right)$, 99.73, 99.53, 99.49, 99.45, 98.90, 98.19, 97.20, 96.03, (C-1 $\left.{ }^{\mathrm{I}-\mathrm{VIII}}\right), 77.76,77.42,(\mathrm{C}-3), 73.59,73.47,73.22$, $70.46,70.25,70.10,69.85,69.31,69.17,68.47,68.24$, $68.10,66.70,66.56,66.42,66.17,65.83,63.25,63.05$, $62.64,62.27,61.90,\left(\mathrm{C}-2,3,4,5,6^{\mathrm{I}-\mathrm{VIII}}\right.$, some signals overlapped); MALDI-TOF MS: Calcd for $\mathrm{C}_{191} \mathrm{H}_{172} \mathrm{O}_{66}$ : $3521.01[\mathrm{M}]$. Found: 3521 [M]. Anal. Calcd for $\mathrm{C}_{191} \mathrm{H}_{172} \mathrm{O}_{66}: \mathrm{C}, 65.11 ; \mathrm{H}, 4.89$. Found: $\mathrm{C}, 65.27 ; \mathrm{H}$, 4.81 .

Allyl $\alpha$-D-mannopyranosyl- $(1 \rightarrow 2)-\alpha$-D-mannopyranosyl- $(1 \rightarrow 3)-\{\alpha$-D-mannopyranosyl- $(1 \rightarrow 2)-\alpha-\mathrm{D}-$ mannopyranosyl- $(1 \rightarrow 3)-[\alpha-\mathrm{D}$ - mannopyranosyl- $(1 \rightarrow 2)-\alpha-\mathrm{D}$ mannopyranosyl-( $(1 \rightarrow 6)]-\alpha$-D-mannopyranosyl- $(1 \rightarrow 6)\}$ $\alpha$-D-mannopyranoside (17). - A saturated solution of ammonia in $\mathrm{MeOH}(5 \mathrm{~mL})$ was added to a solution of 16 (704 mg, $0.2 \mathrm{~mol})$ in $\mathrm{MeOH}(4 \mathrm{~mL})$. After a week at $\mathrm{rt}$, the reaction mixture was concentrated, and the residue was purified by chromatography on Sephadex LH-20 (MeOH) to afford $17(230 \mathrm{mg}, 85 \%)$ as a syrup; ${ }^{1} \mathrm{H}$ NMR $\left(\mathrm{D}_{2} \mathrm{O}\right): \delta 5.87\left(\mathrm{~m}, 1 \mathrm{H}, \mathrm{CH}_{2}=\mathrm{CH}-\mathrm{CH}_{2}\right), 5.31$ $(\mathrm{s}, 1 \mathrm{H}), 5.28\left(\mathrm{dd}, 1 \mathrm{H}, \mathrm{CH}_{2}=\mathrm{CH}-\mathrm{CH}_{2}\right), 5.25(\mathrm{~s}, 1 \mathrm{H})$, $5.20\left(\mathrm{dd}, 1 \mathrm{H}, \mathrm{CH} \mathrm{H}_{2}=\mathrm{CH}-\mathrm{CH}_{2}\right), 5.08(\mathrm{~s}, 1 \mathrm{H}), 4.95$ (s, 3 $\mathrm{H}), 4.80(\mathrm{~s}, 1 \mathrm{H}), 4.60(\mathrm{~s}, 1 \mathrm{H}), 4.05-3.53(\mathrm{~m}, 50 \mathrm{H}) ;{ }^{13} \mathrm{C}$ NMR $\left(\mathrm{D}_{2} \mathrm{O}\right): \delta$ 136.88, 118.54, $\left(\mathrm{CH}_{2}=\mathrm{CH}-\mathrm{CH}_{2}\right)$, $101.98,101.86,101.86,100.56,100.36,100.28,98.99$, 97.60, (C-1 $\left.{ }^{\mathrm{I}-\mathrm{VIII}}\right), 78.44,78.38,(\mathrm{C}-3), 72.75,72.26$, $70.71,70.22,69.85,69.58,69.50,69.08,66.42,66.32$, $66.24,66.03,65.24,65.03,64.65,60.80,60.68,60.48$, 59.96, 58.23, (C-2,3,4,5,6 ${ }^{\mathrm{I}-\mathrm{VIII}}, \mathrm{CH}_{2}=\mathrm{CH}-\mathrm{CH}_{2}$, some signals overlapped); MALDI-TOF MS: Calcd for $\mathrm{C}_{51} \mathrm{H}_{86} \mathrm{O}_{41}: 1354.46$ [M]. Found: 1354.66 [M].

Allyl 2-O-acetyl-3,4,6-tri-O-benzoyl- $\alpha$-D-mannopyranosyl- $(1 \rightarrow 2)$-3,4,6-tri-O-benzoyl- $\alpha$-D-mannopyranosyl - $(1 \rightarrow 3)$ - 2 - O - benzoyl - 4,6 - O - benzylidene- $\alpha$-Dmannopyranoside (22). - Compound 7 was benzoylated with $\mathrm{BzCl}$-pyridine to furnish $\mathbf{2 2}$ as a foamy solid in quantitative yield: $\left.[\alpha]_{\mathrm{D}}-17.7^{\circ}(c) 1.0, \mathrm{CHCl}_{3}\right) ;{ }^{1} \mathrm{H}$ NMR $\left(\mathrm{CDCl}_{3}\right): \delta 8.02-7.33(\mathrm{~m}, 35 \mathrm{H}, \mathrm{Bz}-\mathrm{H}), 6.00(\mathrm{t}, J$ $10.0 \mathrm{~Hz}, 1 \mathrm{H}), 5.87\left(\mathrm{~m}, 1 \mathrm{H}, \mathrm{CH}_{2}=\mathrm{CH}-\mathrm{CH}_{2}-\right), 5.84(\mathrm{dd}$, $J 3.0, J 10.0 \mathrm{~Hz}, 1 \mathrm{H}), 5.78(\mathrm{t}, J 10.0 \mathrm{~Hz}, 1 \mathrm{H}), 5.75(\mathrm{dd}$, $J 2.8, J 10.0 \mathrm{~Hz}, 1 \mathrm{H}), 5.70\left(\mathrm{dd}, J_{2,3} 2.9, J_{1,2} 1.3 \mathrm{~Hz}, 1\right.$ $\mathrm{H}, \mathrm{H}-2), 5.58$ (s, $\left.2 \mathrm{H}, \mathrm{H}-1, \mathrm{H}-2^{\prime \prime}\right), 5.46$ (s, $1 \mathrm{H}, \mathrm{Ph}-\mathrm{CH}$ ), $5.33\left(\mathrm{dd}, 1 \mathrm{H}, \mathrm{CH}_{2}=\mathrm{CH}-\mathrm{CH}_{2}\right), 5.26(\mathrm{dd}, 1 \mathrm{H}$, $\left.\mathrm{CH}_{2}=\mathrm{CH}-\mathrm{CH}_{2}\right), 5.11(\mathrm{~s}, 1 \mathrm{H}), 5.04(\mathrm{~s}, 1 \mathrm{H}),\left(\mathrm{H}-1^{\prime}\right.$, $\left.\mathrm{H}-1^{\prime \prime}\right), 4.67-4.54(\mathrm{~m}, 4 \mathrm{H}), 4.36\left(\mathrm{dd}, J_{2,3} 2.9, J_{1,2} 1.2 \mathrm{~Hz}\right.$, $\left.1 \mathrm{H}, \mathrm{H}-2^{\prime}\right), 4.28(\mathrm{~m}, 1 \mathrm{H}), 4.23-4.17(\mathrm{~m}, 3 \mathrm{H}), 4.08(\mathrm{~m}$, $1 \mathrm{H}), 3.99-3.92(\mathrm{~m}, 3 \mathrm{H}), 3.85\left(\mathrm{t}, J_{4,5} 10.0 \mathrm{~Hz}, 1 \mathrm{H}\right.$, $\mathrm{H}-4), 2.02\left(\mathrm{~s}, 3 \mathrm{H}, \mathrm{CH}_{3} \mathrm{CO}\right) ;{ }^{13} \mathrm{C} \mathrm{NMR}\left(\mathrm{CDCl}_{3}\right): \delta$ $168.68\left(\mathrm{CH}_{3} \mathrm{CO}\right), 165.98,165.63,165.30,165.13,164.67$, 164.67, 164.45, (7 $\left.\mathrm{C}_{6} \mathrm{H}_{5} \mathrm{CO}\right), \quad 136.59, \quad 118.18$, $\left(\mathrm{CH}_{2}=\mathrm{CH}-\mathrm{CH}_{2}-\right)$, 101.51, ( $\left.\mathrm{Ph}-\mathrm{C}\right), 99.15,98.47,97.27$, $\left(\mathrm{C}-1^{\mathrm{I}-\mathrm{III}}\right), 78.74,(\mathrm{C}-3), 75.20,71.67,71.52,70.22,69.25$, $69.06,69.06,69.00,68.34,68.05,66.64,66.29,63.23$, 63.16, 62.16, (C-2,3,4,5,6 $\left.6^{\mathrm{I}-\mathrm{III}}, \mathrm{CH}_{2}=\mathrm{CH}-\mathrm{CH}_{2}-\right)$. Anal. Calcd for $\mathrm{C}_{79} \mathrm{H}_{70} \mathrm{O}_{24}: \mathrm{C}, 67.62 ; \mathrm{H}, 4.99$. Found: C, 67.67; H, 5.01.

Allyl 2-O-acetyl-3,4,6-tri-O-benzoyl- $\alpha$-D-mannopyranosyl-( $1 \rightarrow 2)$ - 3,4,6-tri-O-benzoyl- $\alpha$-D-mannopyranosyl-(1 $\rightarrow 3)$-2-O-benzoyl- $\alpha$-D-mannopyranoside (23).Acetyl chloride $(0.05 \mathrm{~mL}, 0.7 \mathrm{mmol})$ was added dropwise to a solution of the trisaccharide 22 (1402 mg, 1 $\mathrm{mmol})$ in anhyd $\mathrm{MeOH}(50 \mathrm{~mL})$. The solution was sealed in a flask and stirred for $2 \mathrm{~h}$ at $\mathrm{rt}$. The reaction was monitored by TLC until the starting material disappeared, at which time the solution was neutralized with $\mathrm{Et}_{3} \mathrm{~N}$, then concentrated to dryness. The residue was passed through a short silica gel column to give $\mathbf{2 3}$ (1246 mg, 96\%) as foamy solid that was directly used for the further reaction: $[\alpha]_{\mathrm{D}}-11.8^{\circ}\left(c 1.0, \mathrm{CHCl}_{3}\right) ;{ }^{1} \mathrm{H}$ NMR $\left(\mathrm{CDCl}_{3}\right): \delta 8.02-7.33(\mathrm{~m}, 35 \mathrm{H}, \mathrm{Bz}-\mathrm{H}), 5.96-$ 5.80 (m, 4 H, H-4', H-4', $\mathrm{H}-3^{\prime}, \mathrm{CH}_{2}=\mathrm{CH}-\mathrm{CH}_{2}-$ ), 5.71 $\left(\mathrm{dd}, J_{2,3} 3.0, J_{3,4} 10.0 \mathrm{~Hz}, 1 \mathrm{H}, \mathrm{H}-3^{\prime}\right), 5.63\left(\mathrm{dd}, J_{2,3} 2.9\right.$, $\left.J_{1,2} 1.3 \mathrm{~Hz}, 1 \mathrm{H}, \mathrm{H}-2^{\prime \prime}\right), 5.60\left(\mathrm{dd}, J_{2,3} 2.8, J_{1,2} 1.2 \mathrm{~Hz}, 1\right.$ $\mathrm{H}, \mathrm{H}-2), 5.58$ (d, $\left.J_{1,2} 1.2 \mathrm{~Hz}, 1 \mathrm{H}, \mathrm{H}-1\right), 5.30$ (dd, $1 \mathrm{H}$, $\left.\mathrm{CH}_{2}=\mathrm{CH}-\mathrm{CH}_{2}\right), 5.22\left(\mathrm{dd}, 1 \mathrm{H}, \mathrm{CH}_{2}=\mathrm{CH}-\mathrm{CH}_{2}\right), 5.11(\mathrm{~d}$, $\left.1 \mathrm{H}, \mathrm{H}-1^{\prime \prime}\right), 5.04\left(\mathrm{~d}, 1 \mathrm{H}, \mathrm{H}-1^{\prime}\right), 4.59-4.39$ (m, $\left.8 \mathrm{H}\right)$, $4.28\left(\mathrm{t}, J_{4,5} 10.0 \mathrm{~Hz}, 1 \mathrm{H}, \mathrm{H}-4\right), 4.20\left(\mathrm{dd}, J_{2,3} 2.8, J_{3,4}\right.$ $10.0 \mathrm{~Hz}, 1 \mathrm{H}, \mathrm{H}-3), 4.04-3.97(\mathrm{~m}, 3 \mathrm{H}), 3.96$ (dd, $J_{2,3}$ $\left.3.0 \mathrm{~Hz}, 1 \mathrm{H}, \mathrm{H}-2^{\prime}\right), 3.79$ (m, $\left.1 \mathrm{H}\right), 2.05$ (s, $3 \mathrm{H}, \mathrm{CH}_{3} \mathrm{CO}$ ); ${ }^{13} \mathrm{C} \mathrm{NMR}\left(\mathrm{CDCl}_{3}\right): \delta 168.78\left(\mathrm{CH}_{3} \mathrm{CO}\right), 166.21,165.93$, $165.54,165.20,164.97,164.65,164.48,\left(7 \mathrm{C}_{6} \mathrm{H}_{5} \mathrm{CO}\right)$, 133.03, 117.67, $\left(\mathrm{CH}_{2}=\mathrm{CH}-\mathrm{CH}_{2}-\right), 100.38,98.23,96.29$, $\left(\mathrm{C}-1^{\mathrm{I}-\mathrm{III}}\right), 76.84,(\mathrm{C}-3), 74.47,72.05,71.89,70.32,69.09$, $68.95,68.84,68.09,68.01,67.62,67.21,67.05,66.83$, 63.06, 61.86, (C-2,3,4,5,6 $\left.6^{\text {I-III }}, \mathrm{CH}_{2}=\mathrm{CH}-\mathrm{CH}_{2}-\right)$. Anal. 
Calcd for $\mathrm{C}_{72} \mathrm{H}_{66} \mathrm{O}_{23}: \mathrm{C}, 66.56 ; \mathrm{H}$, 5.08. Found: $\mathrm{C}$, 67.47; H, 5.01.

Allyl 2-O-acetyl-3,4,6-tri-O-benzoyl- $\alpha$-D-mannopyranosyl-(1 $\rightarrow 2)$ - 3,4,6-tri-O-benzoyl- $\alpha$-D-mannopyranosyl-(1 $\rightarrow 3)$-4,6-di-O-acetyl-2-O-benzoyl- $\alpha$-D-mannopyranoside (24). - Compound $\mathbf{2 3}$ was acetylated with $\mathrm{Ac}_{2} \mathrm{O}$-pyridine to furnish $\mathbf{2 4}$ as a foamy solid in quantitative yield: $[\alpha]_{\mathrm{D}}-7.6^{\circ}\left(c\right.$ 1.0, $\left.\mathrm{CHCl}_{3}\right) ;{ }^{1} \mathrm{H}$ NMR $\left(\mathrm{CDCl}_{3}\right): \delta 8.02-7.33(\mathrm{~m}, 35 \mathrm{H}, \mathrm{Bz}-\mathrm{H}), 5.95-5.78(\mathrm{~m}, 4$ $\left.\mathrm{H}, \mathrm{H}-4^{\prime}, \mathrm{H}-4^{\prime \prime}, \mathrm{H}-3^{\prime \prime}, \mathrm{CH}_{2}=\mathrm{CH}-\mathrm{CH}_{2}-\right), 5.63\left(\mathrm{dd}, J_{2,3}\right.$ 3.0, $\left.J_{3,4} 10.0 \mathrm{~Hz}, 1 \mathrm{H}, \mathrm{H}-3^{\prime}\right), 5.58$ (s, $\left.2 \mathrm{H}, \mathrm{H}-2, \mathrm{H}-2^{\prime \prime}\right)$, $5.53\left(\mathrm{t}, J_{3,4} 10.0 \mathrm{~Hz}, 1 \mathrm{H}, \mathrm{H}-4\right), 5.37$ (s, $\left.1 \mathrm{H}, \mathrm{H}-1\right), 5.33$ $\left(\mathrm{dd}, \quad 1 \mathrm{H}, \quad \mathrm{CH}_{2}=\mathrm{CH}-\mathrm{CH}_{2}\right), \quad 5.25(\mathrm{dd}, 1 \mathrm{H}$, $\left.\mathrm{CH}_{2}=\mathrm{CH}-\mathrm{CH}_{2}\right), 5.07(\mathrm{~s}, 1 \mathrm{H}, \mathrm{H}-1), 4.99(\mathrm{~s}, 1 \mathrm{H}, \mathrm{H}-1)$, $4.52-4.41(\mathrm{~m}, 7 \mathrm{H}), 4.29-4.15(\mathrm{~m}, 4 \mathrm{H}), 4.04(\mathrm{~m}, 1 \mathrm{H})$, $3.91(\mathrm{~m}, 1 \mathrm{H}), 2.13\left(\mathrm{~s}, 3 \mathrm{H}, \mathrm{CH}_{3} \mathrm{CO}\right), 2.12(\mathrm{~s}, 3 \mathrm{H}$, $\left.\mathrm{CH}_{3} \mathrm{CO}\right), 1.99$ (s, $\left.3 \mathrm{H}, \mathrm{CH}_{3} \mathrm{CO}\right) ;{ }^{13} \mathrm{C}$ NMR $\left(\mathrm{CDCl}_{3}\right): \delta$ $170.19,169.38,168.70,\left(3 \mathrm{CH}_{3} \mathrm{CO}\right), 165.85,165.58$, $165.41,165.16,164.78,164.43,164.43,\left(7 \mathrm{C}_{6} \mathrm{H}_{5} C \mathrm{O}\right)$, 133.18, 118.25, $\left(\mathrm{CH}_{2}=\mathrm{CH}-\mathrm{CH}_{2}-\right), 99.65,99.36,96.09$, (C-1 $\left.{ }^{\mathrm{I}-\mathrm{III}}\right), 76.80,(\mathrm{C}-3), 74.74,71.33,69.71,69.24,69.14$, $69.10,68.94,68.45,68.13,67.42,66.57,66.48,62.94$, 62.82, 62.08, (C-2,3,4,5,6 $\left.{ }^{\mathrm{I}-\mathrm{III}}, \mathrm{CH}_{2}=\mathrm{CH}-\mathrm{CH}_{2}-\right)$. Anal. Calcd for $\mathrm{C}_{76} \mathrm{H}_{70} \mathrm{O}_{25}: \mathrm{C}, 65.99 ; \mathrm{H}, 5.07$. Found: $\mathrm{C}$, 66.17; H, 5.01 .

2-O-Acetyl-3,4,6-tri-O-benzoyl- $\alpha$-D-mannopyranosyl$(1 \rightarrow 2)-3,4,6$-tri-O-benzoyl- $\alpha$-D-mannopyranosyl- $(1 \rightarrow 3)$ 4,6-di-O-acetyl-2-O-benzoyl- $\alpha, \beta$-D-mannopyranose (25). - To a solution of 24 (691 mg, $0.5 \mathrm{mmol})$ in $90 \%$ $\mathrm{AcOH}(10 \mathrm{~mL})$ containing sodium acetate $(293 \mathrm{mg}, 3$ mmol) was added $\mathrm{PdCl}_{2}(89 \mathrm{mg}, 0.5 \mathrm{mmol})$, and the mixture was stirred for $12 \mathrm{~h}$, at the end of which time TLC (2:1 petroleum ether-EtOAc) indicated that the reaction was complete. The mixture was diluted with $\mathrm{CH}_{2} \mathrm{Cl}_{2}(30 \mathrm{~mL})$ and washed with water and satd aq $\mathrm{NaHCO}_{3}$. The organic layer was concentrated under reduced pressure, and the residue thus obtained was passed through a short silica gel column with 2:1 petroleum ether-EtOAc as the eluent to give $\mathbf{2 5}$ as a foamy solid $(604 \mathrm{mg}, 90 \%):[\alpha]_{\mathrm{D}}-29.8^{\circ}$ (c 1.2, $\left.\mathrm{CHCl}_{3}\right) ;{ }^{1} \mathrm{H}$ NMR $\left(\mathrm{CDCl}_{3}\right): \delta \quad 8.02-7.23(\mathrm{~m}, 35 \mathrm{H}$, $\mathrm{Bz}-\mathrm{H}), 5.92(\mathrm{t}, J 10.0 \mathrm{~Hz}, 1 \mathrm{H}), 5.86(\mathrm{t}, J 10.0 \mathrm{~Hz}, 1 \mathrm{H})$, $\left(\mathrm{H}-4^{\prime}, \mathrm{H}-4^{\prime \prime}\right), 5.79(\mathrm{dd}, J 2.8, J 10.0 \mathrm{~Hz}, 1 \mathrm{H}), 5.61(\mathrm{dd}$, $J 2.8, J 10.0 \mathrm{~Hz}, 1 \mathrm{H}),\left(\mathrm{H}-3^{\prime}, \mathrm{H}-3^{\prime \prime}\right), 5.58\left(\mathrm{dd}, J_{2,3} 2.8\right.$, $\left.J_{1,2} 1.2 \mathrm{~Hz}, 1 \mathrm{H}, \mathrm{H}-2\right), 5.55(\mathrm{~s}, 1 \mathrm{H}, \mathrm{H}-1), 5.51\left(\mathrm{t}, J_{3,4}\right.$ $10.0 \mathrm{~Hz}, 1 \mathrm{H}, \mathrm{H}-4), 5.39$ (dd, $J_{2,3} 2.8, J_{1,2} 1.2 \mathrm{~Hz}, 1 \mathrm{H}$, H-2"), 5.36 (s, $\left.1 \mathrm{H}, \mathrm{H}-1^{\prime \prime}\right), 4.99$ (s, $\left.1 \mathrm{H}, \mathrm{H}-1^{\prime}\right), 4.60-4.39$ $(\mathrm{m}, 7 \mathrm{H}), 4.23\left(\mathrm{~m}, 2 \mathrm{H}, \mathrm{H}-3, \mathrm{H}-2^{\prime}\right), 4.16(\mathrm{~m}, 1 \mathrm{H}), 4.05$ $(\mathrm{m}, 1 \mathrm{H}), 2.10\left(\mathrm{~s}, 3 \mathrm{H}, \mathrm{CH}_{3} \mathrm{CO}\right), 2.09\left(\mathrm{~s}, 3 \mathrm{H}, \mathrm{CH}_{3} \mathrm{CO}\right)$, $1.96\left(\mathrm{~s}, 3 \mathrm{H}, \mathrm{CH}_{3} \mathrm{CO}\right) ;{ }^{13} \mathrm{C} \mathrm{NMR}\left(\mathrm{CDCl}_{3}\right): \delta 170.32$, 169.44, 168.73, (3 $\left.\mathrm{CH}_{3} \mathrm{CO}\right), 165.90,165.73,165.40$, $165.19,164.81,164.52,164.44,\left(7 \mathrm{C}_{6} \mathrm{H}_{5} \mathrm{CO}\right), 99.46$, 99.44, 91.71, (C-1 $\left.{ }^{\mathrm{I}-\mathrm{III}}\right), 76.70,(\mathrm{C}-3), 73.98,71.51,69.66$, $69.42,69.24,69.15,68.97,68.39,67.37,66.68,66.57$, $63.03,62.87,62.15,\left(\mathrm{C}-2,3,4,5,6^{\mathrm{I}-\mathrm{III}}\right)$. Anal. Calcd for $\mathrm{C}_{73} \mathrm{H}_{66} \mathrm{O}_{25}: \mathrm{C}, 65.28 ; \mathrm{H}, 4.92$. Found: $\mathrm{C}, 65.07 ; \mathrm{H}, 5.01$.
2-O-Acetyl-3,4,6-tri-O-benzoyl- $\alpha$-D-mannopyranosyl$(1 \rightarrow 2)-3,4,6$-tri-O-benzoyl- $\alpha$-D-mannopyranosyl- $(1 \rightarrow 3)$ 4,6-di-O-acetyl-2-O-benzoyl- $\alpha$-D-mannopyranosyl trichloroacetimidate (26). - Compound 25 (537 mg, 0.4 mmol) was dissolved in $\mathrm{CH}_{2} \mathrm{Cl}_{2}(20 \mathrm{~mL})$, then $\mathrm{CCl}_{3} \mathrm{CN}$ $(0.1 \mathrm{ml}, 2 \mathrm{mmol})$ and DBU $(14 \mu \mathrm{L}, 0.18 \mathrm{mmol})$ were added. The reaction mixture was stirred for $2 \mathrm{~h}$, at the end of which time TLC (2:1 petroleum ether-EtOAc) indicated that the reaction was complete. Concentration of the reaction mixture, followed by purification on a silica gel column with $2: 1$ petroleum ether-EtOAc as the eluent furnished $\mathbf{2 6}$ as crystals in good yield (535 $\mathrm{mg}, 90 \%):{ }^{1} \mathrm{H}$ NMR $\left(\mathrm{CDCl}_{3}\right): \delta 8.79(\mathrm{~s}, 1 \mathrm{H}, \mathrm{N}=\mathrm{H})$, 8.02-7.23 (m, $35 \mathrm{H}, \mathrm{Bz}-\mathrm{H}), 6.52(\mathrm{~s}, 1 \mathrm{H}, \mathrm{H}-1), 5.95(\mathrm{t}$, $J 10.0 \mathrm{~Hz}, 1 \mathrm{H}), 5.89$ (t, $J 10.0 \mathrm{~Hz}, 1 \mathrm{H}),\left(\mathrm{H}-4^{\prime}, \mathrm{H}-4^{\prime \prime}\right)$, 5.79-5.75 (m, $\left.2 \mathrm{H}, \mathrm{H}-2, \mathrm{H}-3^{\prime \prime}\right), 5.66\left(\mathrm{t}, J_{3,4} 10.0 \mathrm{~Hz}, 1\right.$ $\mathrm{H}, \mathrm{H}-4), 5.62\left(\mathrm{dd}, J_{2,3} 3.0, J_{3,4} 10.0 \mathrm{~Hz}, 1 \mathrm{H}, \mathrm{H}-3^{\prime}\right), 5.56$ (dd, $\left.J_{2,3} 2.8, J_{1,2} 1.2 \mathrm{~Hz}, 1 \mathrm{H}, \mathrm{H}-2^{\prime \prime}\right), 5.38$ (s, $1 \mathrm{H}, \mathrm{H}-1^{\prime \prime}$ ), $5.03\left(\mathrm{~s}, 1 \mathrm{H}, \mathrm{H}-1^{\prime}\right), 4.52-4.35(\mathrm{~m}, 7 \mathrm{H}), 4.25-4.18(\mathrm{~m}$, $4 \mathrm{H}), 2.13$ (s, $3 \mathrm{H}, \mathrm{CH}_{3} \mathrm{CO}$ ), 2.08 (s, $\left.3 \mathrm{H}, \mathrm{CH}_{3} \mathrm{CO}\right), 1.96$ (s, $3 \mathrm{H}, \mathrm{CH}_{3} \mathrm{CO}$ ). Anal. Calcd for $\mathrm{C}_{75} \mathrm{H}_{66} \mathrm{Cl}_{3} \mathrm{NO}_{25}$ : C, 60.52; H, 4.44. Found: C, 60.37; H, 4.51.

Methyl 2-O-acetyl-3,4,6-tri-O-benzoyl- $\alpha$-D-mannopyranosyl- $(1 \rightarrow 2)$ - 3,4,6-tri-O-benzoyl- $\alpha$-D-mannopyranosyl-( $1 \rightarrow 3)-4,6$-di-O-acetyl-2-O-benzoyl- $\alpha$-D-mannopyranosyl- $(1 \rightarrow 2)-3,4,6$ - tri-O - benzoyl- $\alpha$-D - mannopyranosyl- $(1 \rightarrow 2)$ - 3,4,6-tri-O-benzoyl- $\alpha$-D-mannopyranosyl-(1 $\rightarrow 2)$-3,4,6-tri-O-benzoyl- $\alpha$-D-mannopyranoside (27). - Compounds 26 (446 mg, $0.3 \mathrm{mmol}$ ) and 21 (437 $\mathrm{mg}, 0.3 \mathrm{mmol})$ were dried together under high vacuum for $2 \mathrm{~h}$, then dissolved in anhyd $\mathrm{CH}_{2} \mathrm{Cl}_{2}(50 \mathrm{~mL})$. TMSOTf ( $5 \mu \mathrm{L}, 0.08$ equiv) was added dropwise at $-20{ }^{\circ} \mathrm{C}$ with $\mathrm{N}_{2}$ protection. The reaction mixture was stirred for $2 \mathrm{~h}$, during which time the reaction temperature was gradually raised to ambient temperature. Then the mixture was neutralized with triethylamine and concentrated under reduced pressure to an oily residue. Purification by column chromatography (1:1 petroleum ether-EtOAc) gave $27(545 \mathrm{mg}, 65 \%)$ as a colorless foamy solid: $[\alpha]_{\mathrm{D}}-17.1^{\circ}\left(c\right.$ 1.0, $\left.\mathrm{CHCl}_{3}\right) ;{ }^{1} \mathrm{H} \mathrm{NMR}$ $\left(\mathrm{CDCl}_{3}\right): \delta 8.19-7.24(\mathrm{~m}, 80 \mathrm{H}, \mathrm{Bz}-\mathrm{H}), 6.06(\mathrm{t}, J 10.0$ $\mathrm{Hz}, 1 \mathrm{H}), 6.00-5.75(\mathrm{~m}, 10 \mathrm{H}), 5.64-5.60(\mathrm{~m}, 2 \mathrm{H})$, $5.53(\mathrm{~s}, 1 \mathrm{H}), 5.40(\mathrm{~s}, 2 \mathrm{H}), 5.29(\mathrm{~s}, 1 \mathrm{H}), 5.10(\mathrm{~s}, 1 \mathrm{H})$, 5.06 (s, 1 H), (six H-1), 4.65-4.21 (m, $18 \mathrm{H}), 4.17-4.12$ $(\mathrm{m}, 2 \mathrm{H}), 4.00(\mathrm{~m}, 1 \mathrm{H}), 3.90(\mathrm{~m}, 1 \mathrm{H}), 3.34(\mathrm{~s}, 3 \mathrm{H}$, $\left.\mathrm{OCH}_{3}\right), 2.04$ (s, $\left.3 \mathrm{H}, \mathrm{CH}_{3} \mathrm{CO}\right), 1.97$ (s, $3 \mathrm{H}, \mathrm{CH}_{3} \mathrm{CO}$ ), $1.94\left(\mathrm{~s}, 3 \mathrm{H}, \mathrm{CH}_{3} \mathrm{CO}\right) ;{ }^{13} \mathrm{C} \mathrm{NMR}\left(\mathrm{CDCl}_{3}\right): \delta 170.80$, $169.90,169.17$, (3 $\left.\mathrm{CH}_{3} \mathrm{CO}\right), 166.64,166.56,166.33$, $166.28,166.20,166.09,165.88,165.77,165.66,165.50$, $165.42,165.38,165.31,165.24,164.96,164.75$, (16 $\left.\mathrm{C}_{6} \mathrm{H}_{5} \mathrm{CO}\right), 100.21,100.04,99.90$, 99.90, 99.51, 99.51, $\left(\mathrm{C}-1^{\mathrm{I}-\mathrm{VI}}\right), 76.66,(\mathrm{C}-3), 72.09,72.00,71.90,71.72,71.46$, $70.68,70.60,69.78,69.78,69.66,69.66,69.56,69.50$, $68.77,68.22,68.22,67.90,67.90,67.07,67.21,67.09$, $66,55,64.09,63.86,63.86,63.72,63.54,63.07,62.95$, $62.88,62.49,62.40,\left(\mathrm{C}-2,3,4,5,6^{\mathrm{I}-\mathrm{VI}}\right)$. Anal. Calcd for 
$\mathrm{C}_{155} \mathrm{H}_{134} \mathrm{O}_{50}: \mathrm{C}, 66.57 ; \mathrm{H}, 4.80$. Found: C, 66.42; H, 4.91 .

Methyl $\alpha$-D-mannopyranosyl- $(1 \rightarrow 2)-\alpha$-D-mannopyranosyl-( $1 \rightarrow 3)$ - $\alpha$-D-mannopyranosyl $(1 \rightarrow 2)-\alpha$-D-mannopyranosyl - $(1 \rightarrow 2)$ - $\alpha$ - D - mannopyranosyl - $(1 \rightarrow 2)-\alpha-\mathrm{D}-$ mannopyranoside (28). - A solution of $27(279 \mathrm{mg}, 0.1$ $\mathrm{mmol})$ in $\mathrm{MeOH}(4 \mathrm{~mL})$ was added to a saturated solution of ammonia in $\mathrm{MeOH}(5 \mathrm{~mL})$. After a week at $\mathrm{rt}$, the reaction mixture was concentrated, and the residue was purified by chromatography on Sephadex LH-20 $(\mathrm{MeOH})$ to afford $28(85 \mathrm{mg}, 85 \%)$ as a syrup: ${ }^{1} \mathrm{H}$ NMR $\left(\mathrm{D}_{2} \mathrm{O}\right): \delta 5.30(\mathrm{~s}, 1 \mathrm{H}), 5.20(\mathrm{~s}, 2 \mathrm{H}), 4.94(\mathrm{~s}$, $1 \mathrm{H}), 4.92(\mathrm{~s}, 1 \mathrm{H}), 4.89$ (s, $1 \mathrm{H}), 3.97-3.50(\mathrm{~m}, 36 \mathrm{H})$, $3.29\left(\mathrm{~s}, 3 \mathrm{H}, \mathrm{OCH}_{3}\right)$. MALDI-TOF MS: Calcd for $\mathrm{C}_{37} \mathrm{H}_{64} \mathrm{O}_{31}: 1004.34$ [M]. Found: 1004.46 [M].

\section{Acknowledgements}

This work was supported by The Chinese Academy of Sciences (Projects KJ952J $\mathrm{J}_{1} 510$ and KIPRCEES9904) and by The National Natural Science Foundation of China (Projects 29802009, 39970864, and 30070815).

\section{References}

1. (a) Moingeon, P.; Chang, H. C.; Sayre, P. H.; Klayton, L. K.; Alcover, A.; Gardner, P. I.; Reinherz, E. L. Immunol. Rev. 1989, 4, 111-144; (b) Siliciano, R. F.; Pratt, J. C.; Schmidt, R. E.; Ritz, J.; Reinherz, E. L. Nature 1985, 317, 423-428;

(c) Yang, S. Y.; Chouaib, S.; Dupont, B. J. Immunol. 1986, 137, 1097-1102;

(d) Hunig, T.; Tiefenthaler, G.; Meyer zum Büschenfelde, K.-H.; Meuer, S. C. Nature 1987, 326, 298-303;

(e) Qin, L.; Chaving, K. D.; Lin, J.; Yagita, H.; Bromberg, J. S. J. Exp. Med. 1994, 179, 341-349.

2. Wyss, D. F.; Choi, J. S.; Li, J.; Knoppers, M. H.; Willis, K. J.; Arulanandam, A. R. N.; Smolyar, A; Reinherz, E. L.; Wagner, G. Science 1995, 269, 1273-1298.

3. Recny, M. A.; Luther, M. A.; Knoppers, M. H.; Neidhardt, E. A.; Khandekar, S. S.; Concino, M. F.; Shimke, P. A.; Francis, M. A.; Moebius, U. J. Biol. Chem. 1992, 267, 22428-22434.

4. Arulanandam, A. R. N.; Smolyar, A; Reinherz, E. L.; Wagner, G. Proc. Natl. Acad. Sci. USA 1993, 90, $11613-$ 11620.

5. Bewley, C. A.; Otero-Quintero, S. J. Am. Chem. Soc. 2001, 123, 3892-3902.

6. Funayama, M.; Nishikawa, A.; Shinoda, T.; Fukazawa, Y. Carbohydr. Res. 1983, 117, 229-239.

7. (a) Wang, W.; Kong, F. J. Org. Chem. 1998, 63, 57445745 ;

(b) Wang, W.; Kong, F. Angew. Chem., Int. Ed. Engl. 1999, 38, 1247-1250.

8. (a) Wang, W.; Kong, F. J. Org. Chem. 1999, 64, 50915095;

(b) Wang, W.; Kong, F. Carbohydr. Res. 1999, 315, 117-126;

(c) Wang, W.; Kong, F. Tetrahedron Lett. 1998, 39, 1937-1940.

9. Zhu, Y.; Kong, F. Synlett 2000, 663-667.

10. Zhu, Y.; Kong, F. Synlett 2000, 1783-1788.

11. Wang, W.; Kong, F. J. Carbohydr. Chem. 1999, 18, 264-272.

12. Yang, G.; Kong, F. Synlett 2000, 1427-1430.

13. Unverzagt, C. Angew. Chem., Int. Ed. Engl. 1994, 33, $1102-1105$. 